\title{
Additive Representations on Rank-Ordered Sets. I. The Algebraic Approach
}

\author{
Peter Wakker
}

University of Nijmegen

\begin{abstract}
This paper considers additive conjoint measurement on subsets of Cartesian products containing "rank-ordered' $n$-tuples. Contrary to what has often been thought, additive conjoint measurement on subsets of Cartesian products has characteristics different from additive conjoint measurement on full Cartesian products. 1991 Academic Press, Inc.
\end{abstract}

\section{History, Motivation, and Preview}

In Section 6.13 of Krantz, Luce, Suppes, \& Tversky (1971) (hereafter abbreviated KLST) three open problems of additive conjoint measurement were formulated. The second problem, regarding additive conjoint measurement on subsets of Cartesian products, is the topic of this paper. Following KLST we use an algebraic setup, i.e., as technical conditions we assume restricted solvability and (versions of) the Archimedean axiom. ${ }^{1}$ Section 6.5.5 of KLST points out the importance of additive conjoint measurement on subsets of Cartesian products. Interest has increased during the last decade because of new developments in the literature on decision making under risk/uncertainty. There independence is often required to hold only within certain subsets.

The following phenomena, typical for subsets of Cartesian products, deviate from the classical results on full Cartesian products.

1. The traditional axioms of additive conjoint measurement do not necessarily imply additive representability if the equivalence classes are not "connected." This holds even if the domain is "full-dimensional" and connected: see Remark III.7.8 in Wakker (1989b). Figure 2 in Wakker (1990e) uses a domain that is rich enough to satisfy restricted solvability.

2. Another reason that the traditional axioms need not imply additive representability is that, loosely speaking, an additive representing function may be

Reprint requests should be sent to Peter Wakker, University of Nijmegen (NICI), Psychological I ahoratory, P.O. Box 9104, 6500 HF Nijmegen, The Netherlands. The research has been made possible by a fellowship of the Royal Netherlands Academy of Arts and Sciences, and a fellowship of the Netherlands Organization for Scientific Research.

${ }^{1}$ Building on this paper, Wakker (1990e) gives results for the topological setup. Wakker (1988) gave arguments in favor of the algebraic setup. 
"driven to $\infty$ or $-\infty$ " at boundary points. This is shown below in Theorem 3(i), and Examples 25b and 25c.

3. Uniqueness results can differ from the prevailing ordinal/interval scales, contrary to claims in the litcraturc. In Examplc 25a additive representations can bc increased arbitrarily at maximal extreme alternatives, and can be decreased arbitrarily at minimal extreme alternatives. In Example 26 the additive representation is an ordinal scale, and each separate additive value function is an ordinal ( = interval there) scale independent of the other additive value functions.

The most important cases of subsets of Cartesian products are presently the rankordered subsets; these are the topic of the present paper. The reason for their importance is that many current approaches in decision making under risk/uncertainty allow probabilities, or transforms thereof, to depend on the ranking of the associated outcomes. Thus the usual additive representability only applies to "comonotonic" subsets, i.e., subsets of alternatives (acts, distributions, ..., that have a fixed ordering of outcomes. Many authors independently developed these rankdependent models in the last decade. Examples from the psychological literature are

[1] Luce (1988) (extending Luce \& Narens, 1985); Formulas 7a, 7b, and 8 in the former are equivalent to Choquet expected utility as described in Wakker (1989b, Formulas VI.2.7 and VI.2.11, and in the text above the latter formula). The original term "dual bilinear" has now been changed to "rank-dependent," in accordance with economic terminology.

Examples from the economic literature are

[2] Quiggin (1982) (decision making under risk),

[3] Yaari (1987) (decision making under risk),

[4] Allais (1988) (decision making under risk),

[5] Schmcidler (1989, first version 1982) (dccision making under uncertainty),

[6] Weymark (1981) (measurement of inequality/welfare theory).

In fuzzy set theory we found

[7] Yager (1988) (multiattribute utility theory),

[8] Murofushi \& Sugeno (1989) (referring to the early Höhle (1982) for the Choquet-integral idea).

The following references build on (some of) the above ones, as indicated by square brackets. For decision making under risk: Miyamoto (1988; [1]); Chew (1989; [2, 3, 6]), Chew \& Epstein (1989a, 1989b; [2, 3, 6]), Green \& Jullien (1988; $[2,3])$, Segal $(1989 ;[2,3]),{ }^{2}$ and many others building on these works. For decision making under uncertainty: Gilboa $(1987$; [5]), Wakker $(1989 \mathrm{a}, 1989 \mathrm{~b}$ Chapter VI, 1989c (extending to infinite state spaces); [5]), Becker \& Sarin (1989; [5]), Chateauneuf (1990a; [5]), Nakamura (1990a; [5]). For welfare theory or the

\footnotetext{
${ }^{2}$ Theorem 1 in Segal (1989) is incorrect, hence the correctness of Theorem 2 is an open question.
} 
measurement of inequality: Ebert (1988a, 1988b; [6]). For dynamic decision making: Gilboa (1989; [5]). For fuzzy set theory: Wakker (1990a; [5]). Wakker $(1990 \mathrm{~b})$ pointed out that rank-dependence under risk is a special case of rank-dependence under uncertainty. The earliest of the above references, Weymark (1981), was brought to our attention by Chew \& Epstein (1989a). We inferred from the text below their Theorem 1 that Weymark's Theorem 3 is a predecessor of Yaari (1987). Also Chew \& Epstein mention the carlicr use of L-estimators in robust statistics. L-estimators are linear combinations of rank-ordered random variables, thus are special cases of (linear-utility) rank-dependent forms analogous to those characterized in Weymark (1981) and Yaari (1987).

Several of the above references already used additive conjoint measurement on rank-ordered subsets. But the intricacies of the topic have not been well understood. Example 25 below provides a counterexample to Chew \& Epstein (1989b, Theorem A), Green \& Jullien (1988, Lemma in the Appendix), Quiggin (1989, Proposition 4), and Nakamura (1990a, Lemma 2). ${ }^{3}$ The problem illustrated by the example is not very fundamental. (Wakker (1990e) comments on incorrect methods of proofs.) Theorem 4(c) below shows that the aforementioned results of Green \& Jullien and Quiggin can be corrected by addition of the strong Archimedean axiom; note that the algebraic setup of the current paper includes as special case the topological setups of the mentioned papers. Corollary 6 (plus Remark 23) below shows that Lemma 2 in Nakamura (1990a) can be straightforwardly corrected, and that his main results are correct. Theorem 4(a) below supplements the proofs of Ebert (1988a, p. 162, 1. 4/5, and 1988b, Theorem 3). The essential techniques of the proof in this paper were already used in Wakker $(1989 a, 1989 b)$. Related methods of proof have been developed, independently, in Nakamura (1990a), and in Miyamoto (1988) for the case of two coordinates. Wakker (1986) may be the earliest work using this method of proof. The three references just mentioned all consider specific setups where the additive value functions are proportional (compare Corollary 6 below). Hence, general additive conjoint measurement results are not easily distilled from those proofs. Recently in Segal (1990), for the case of Euclidean spaces, results have becn given for open connected subsets that have all indifference surfaces, and all intersections with hyperplanes perpendicular to an axis, connected. This includes interiors of rank-ordered sets as a special case. Also, a condition is given to exclude the "driven to $\infty$ or $-\infty$ " phenomenon in special cases (any "extreme coordinate" occurs in three alternatives where the first strictly dominates the second, and the second the third, on each other coordinate). These cases do not include all boundary points in rank-ordered sets, in particular not the extreme alternatives. Doignon \& Falmagne (1974) studied difference measurement, i.e., additive conjoint measurement for two dimcnsions where the second additive value function is minus the first, on subsets of Cartesian products. One difference with this paper is that difference measurement satisfies a "reversed" monotonicity

\footnotetext{
${ }^{3}$ Wakker (1990f, Example 3) gives an analogous infinite-dimensional counterexample to Theorem 1 in Segal (1989).
} 
condition. Additive representation on subsets of Cartesian products when probability distributions over alternatives are available, and are evaluated by expected utility, have been studied in Fishburn (1976), and the references therein. For Cartesian products containing finitely many elements, necessary and sufficient conditions are known for additive representability, that apply as well to subsets of Cartesian products. See KLST (Chapter IX), Scott (1964), and many other references. These were extended to necessary and sufficient conditions for arbitrary countable subsets of Cartesian products in Jaffray (1974b, for two dimensions; 1974a, for arbitrary finite dimensions); the latter also gave necessary and sufficient conditions for uncountable full Cartesian products, as well as suggestions for uncountable subsets.

This paper is organized as follows. In Subsection 2.1 the set of rank-ordered alternatives is introduced. Subsection 2.2 gives technical definitions, including a new Archimedean axiom. Subsection 2.3 gives the most important intuitive conditions: $\mathrm{CI}$ and generalized triple cancellation. Subsection 2.4 gives the main results. The major part of the proof of the main results is carried out in Section 3. Section 4 completes the proofs. Section 5 gives elucidations.

\section{The Main Result}

\subsection{Rank-Ordered Alternatives; Extreme Alternatives}

Let $\mathscr{C}$ be a nonempty set, with elements called outcomes. Let $\geqslant^{\prime}$ be a weak order on $\mathscr{C}$, with $\succ^{\prime}, \preccurlyeq^{\prime},\left\langle^{\prime}, \sim^{\prime}\right.$ as usual. Let $n \geqslant 2$. An $n$-tuple $\left(x_{1}, \ldots, x_{n}\right) \in \mathscr{C}^{n}$ is rankordered if $x_{1} \geqslant \cdots \geqslant x_{n}$, and is then called a rank-ordered alternative. The set of rank-ordered alternatives is denoted as $\mathscr{C}_{r}^{n}$. See Fig. 1. Since non-rank-ordered alternatives are not considered in this paper, we simply say alternative instead of rank-ordered alternative.

An outcome $\alpha$ is maximal if $\beta \succ^{\prime} \alpha$ for no outcome $\beta, \alpha$ is minimal if $\beta<^{\prime} \alpha$ for

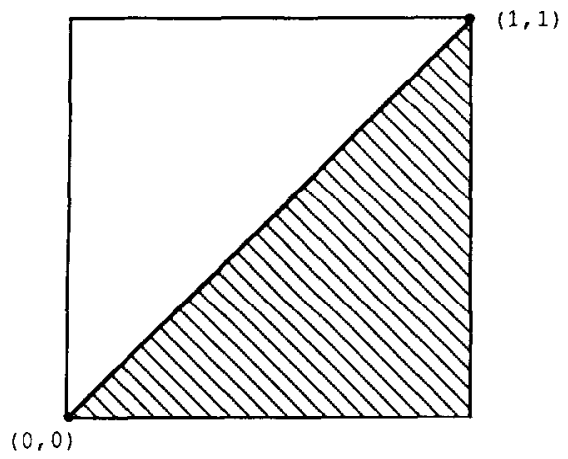

FIG. 1. Let $n=2, \mathscr{C}=[0,1] . \mathscr{C}_{r}^{2}$ is the dashed area, i.e. $\left\{\left(x_{1}, x_{2}\right): x_{1} \geqslant x_{2}\right\}$. 
no outcome $\beta$. Note that a rank-ordered alternative with maximal $n$th coordinate must have all of its coordinates maximal, and a rank-ordered alternative with minimal first coordinate must have all of its coordinates minimal. We call such alternatives extreme. We shall see in this paper, where the set under consideration is a rankordered subset of a Cartesian product, that complications and new phenomena can occur for extreme alternatives.

We consider a binary (preference) relation $\geqslant$ on a subset $X$ of $\mathscr{C}_{r}^{n} . X$ will contain all alternatives except the extreme ones in, firstly, Theorem 4(b), secondly, the proof of Theorem 4(b) in Section 3 and Subsection 4.1, and, thirdly, in part of Example 26. In the remainder of the paper the extreme alternatives will be included. The binary relations $\succ, \preccurlyeq, \prec, \sim$ will be as usual. Often a "constant" alternative $(\alpha, \ldots, \alpha)$ will be identified with the outcome $\alpha \in \mathscr{C}$. Transitivity, completeness, and the monotonicity assumption in this paper will guarantee that $\geqslant^{\prime}$ and $\geqslant$ are in agreement, i.e., $(\alpha, \ldots, \alpha)>(\beta, \ldots, \beta) \Rightarrow \alpha \succ^{\prime} \beta,(\alpha, \ldots, \alpha) \sim(\beta, \ldots, \beta) \Rightarrow \alpha \sim^{\prime} \beta$. Hence we will often omit the accent, and write for instance $\alpha \geqslant \beta$ instead of $\alpha \geqslant{ }^{\prime} \beta$. The tricky introduction with $\succcurlyeq^{\prime}$ was necessary since prior to defining $\geqslant$ we needed the set $X$, but prior to defining rank-orderedness and the set $X$ we already needed $\geqslant$, the ordering of outcomes in agreement with the ordering of constant alternatives.

\subsection{Technical Definitions of Additive Conjoint Measurement}

In this subsection we give technical definitions, all adapted to arbitrary subsets $E$ of Cartesian products. If $E$ is the entire domain $X$ of $\geqslant$, then "on $E$ " is often omitted below. $\overline{\mathbb{R}}:=\mathbb{R} \cup\{-\infty, \infty\}$ is endowed with the usual addition and multiplication rules, and ordering; $\infty-\infty$ is undefined and will not occur.

Throughout, let $E \subset \mathscr{C}_{r}^{n}$. $\succcurlyeq$ is a weak order on $E$ if it is transitive on $E$ $(\forall x, y, z \in E:[x \geqslant y, y \geqslant z] \Rightarrow[x \geqslant z])$ and complete $(\forall x, y \in E: x \geqslant y$ or $y \geqslant x)$. A function $V$ represents $\geqslant$ on $E$ if $E$ is contained in the domain of $V$, $V$ 's range is $\mathbb{R}$, and $x \geqslant y \Leftrightarrow V(x) \geqslant V(y)$. A function $V$ is additive on $E$ if, on $E$, $V: x \mapsto \sum_{j=1}^{n} V_{j}\left(x_{j}\right)$ for some functions $V_{1}, \ldots, V_{n}$ to $\mathbb{R}$; the $V_{j}$ 's must obviously contain the projections of $E$ in their domain. If an additive function represents $\geqslant$, then the $V_{j}$ 's are called additive value functions. A function $V$ is an interval scale (or "cardinal") if, loosely speaking, it "can be replaced" by any function $\tau+\sigma V$ for real $\tau$ and positive $\sigma$. If an additive function is an interval scale, then the additive value functions will be "joint interval scales"; i.e., varying arbitrary real numbers ("locations") can be added to each of them, and they can be multiplied by a joint positive factor ("scale"). The above two uniqueness results correspond to each other, we shall usually make explicit the result applying to the additive function.

If in the above definitions the range of $V$ (and $V$, s) is $\overline{\mathbb{R}}$ instead of $\mathbb{R}$, then $V$ is called an extended representation, rcspcctivcly extended additive representation, and the $V_{j}$ 's are called extended additive value functions. It may occur in this paper that one additive value function contains $\infty$ in its range, another $-\infty$. The rankordered shape of the domain of the additive representation will then guarantee that never $\infty-\infty$ will occur in evaluations of alternatives.

We write $x_{-i} \alpha$ for $x$ with $x_{i}$ replaced by $\alpha$, and, for $i \neq j, x_{-i, j} \alpha, \beta$ for $x$ with $x_{i}$ 
replaced by $\alpha, x_{j}$ replaced by $\beta$. "For all $x_{-i} \alpha$ (such that...)" is short for "for all $i$, $x, \alpha$ (such that $x_{-i} \alpha \ldots$..." If some of the involved variables have already been defined then no quantification over them should be taken; if this is clear from the context we do not make it explicit. The same remarks apply to "there exists" as to "for all."

$\geqslant$ satisfies restricted solvability on $E$ if, for all $x_{-i} \alpha, y, x_{-i} \gamma \in E$ :

$$
x_{-i} \alpha>y>x_{-i} \gamma \Rightarrow \exists x_{-i} \beta \in E: x_{-i} \beta \sim y .
$$

Coordinate $i$ is inessential on $E$ (with respect to $\geqslant$ ) if $\left[\forall x, x_{-i} \alpha \in E: x \sim x_{-i} \alpha\right]$. The opposite of inessential is essential.

The binary relation $\geqslant$ satisfies strong monotonicity, or monotonicity for short, on $E$ if

$$
\alpha \geqslant \beta \Leftrightarrow x_{-i} \alpha \geqslant x_{-i} \beta
$$

for all $x_{-i} \alpha, x_{-i} \beta \in E$. Since the only version of monotonicity occurring in this paper will be strong monotonicity, the adjective "strong" is usually omitted. On sets $E$ that are "rich enough," monotonicity implies that all coordinates are essential. Analogously a function $V$ satisfies strong monotonicity, or monotonicity for short, on $E$ if

$$
\alpha \geqslant B \Leftrightarrow V\left(x_{-i} \alpha\right) \geqslant V\left(x_{-i} \beta\right)
$$

for all $x_{-i} \alpha, x_{-i} \beta \in E$.

Next we adapt the traditional Archimedean axiom of KLST to rank-ordered subsets, analogously to Miyamoto (1988). As a preparation we define a standard sequence; a standard sequence may be infinite or finite of any length. For coordinate $j$ we call $\alpha^{1}, \alpha^{2}, \ldots$, a standard sequence (on coordinate $j$ ) with respect to $\geqslant$ on $E \subset \mathscr{C}^{n}$, if there exist $x, i \neq j$, and $v_{i} \not w_{i} \in \mathscr{C}$, such that $x_{-i, j} v_{i}, \alpha^{k} \sim x_{-i, j} w_{i}, \alpha^{k+1}$ for all $k=1,2, \ldots$, with furthermore all involved alternatives contained in $E$. A standard sequence on coordinate $j$ is bounded (on $E$ ) if there exist $\alpha^{\text {sup }}$ and $\alpha^{\text {inf }}$ such that $\alpha^{\text {sup }} \geqslant \alpha^{k} \geqslant \alpha^{\text {inf }}$ for all $k$ with $\alpha^{\text {sup }}=x_{j}, \alpha^{\text {inf }}=y_{j}$ for some $x, y \in E$. The Archimedean axiom requires that every bounded standard sequence is finite. This is obviously necessary for additive (real-valued) representability, since the preferences involved in a standard sequence imply that the differences $V_{j}\left(\alpha^{k+1}\right)-V_{j}\left(\alpha^{k}\right)$ are constant. We show that on rank-ordered subsets this traditional Archimedean axiom is not strong enough to guarantee that additive representations are realvalued. The reason is that the structure is not rich enough to reveal all the required equalities of $V_{j}$-differences; see Fig. 4. One might strengthen the Archimedean axiom by considering, more generally, standard sequences derived from equivalences $x_{-j} \alpha^{k} \sim y_{-j} \alpha^{k+1}$ (with not $x_{-j} \alpha^{1} \sim y_{-j} \alpha^{1}$ ), for all $k=1,2, \ldots$, with again all involved alternatives contained in $E$. Both on full Cartesian products, and on rankordered subsets, the resulting Archimedean axiom turns out to be equivalent to the one above in the presence of the other conditions. So it does not give the desired strengthening. On a rank-ordered subset the following strengthening will suffice to reveal all required inequalities of $V_{r}$-differences. For coordinate $j$ we call $\alpha^{1}, \alpha^{2}, \ldots$, 
a second-order accelerating sequence (on coordinate $j$ ) with respect to $\geqslant$ on $E \subset \mathscr{C}^{n}$, if for every $k$ there exists a standard sequence $\left(\beta^{1}, \beta^{2}, \ldots\right)$ on coordinate $j$ with respect to $\geqslant$ on $E$, such that either, for some $m, m^{\prime}, l \in \mathbb{N}, \beta^{m} \preccurlyeq \alpha^{k} \preccurlyeq \alpha^{k+1} \preccurlyeq \beta^{m+l}$ and $\alpha^{k+1} \preccurlyeq \beta^{m^{\prime}} \preccurlyeq \beta^{m^{\prime}+1} \preccurlyeq \alpha^{k+2}$, or this holds with everywhere $\succcurlyeq$ instead of $\preccurlyeq$. We might use the term "second-order standard sequence" if we would put equivalences instead of the two extreme preferences in both triples of preferences above. Using second-order standard sequences instead of second-order accelerating sequences in the Archimedean axiom below would make it too weak; this can be inferred from an analogue of Example III.6.8b in Wakker $(1989 \mathrm{~b})$. In the topological setup it will suffice though; see Wakker (1990e). The strong Archimedean axiom requires that every bounded second-order accelerating sequence is finite. We have abstained from the more accurate, but intractable, term "second-order acceleration Archimedean axiom." Since any standard sequence is a second-order accelerating sequence, the strong Archimedean axiom implies the usual one. Again the axiom is necessary for additive representability: under additive representability the absolute value of $V_{j}$-differences of subsequent outcomes in a second-order accelerating sequence is nondecreasing. Unfortunately the condition is complicated and not very appealing. It is one step closer to the general, but very complicated, conditions for additive representability as provided in Jaffray $(1974 a, 1974 b)$. Still, since there is great interest in additive representation on rank-ordered subsets nowadays, and since the condition allows the correction of several results in the literature, we have decided to present it.

\subsection{Intuitive Axioms for Additive Conjoint Measurement}

In this subsection we present the most important characterizing conditions, CI and generalized triple cancellation, two special cases of the "cancellation axioms" of KLST. For $n=2$ the condition below is identical to triple cancellation as in KLST. The present formulation of triple cancellation allows the derivation of a general theorem, applying simultaneously to all $n \geqslant 2$.

DefinItion 1. We say $\succcurlyeq$ satisfies generalized triple cancellation on $E \subset \mathscr{C}^{n}$ if

$$
\begin{aligned}
& x_{-i} \alpha \preccurlyeq y_{-i} \beta \quad \& \quad v_{-i} \alpha \geqslant w_{-i} \beta \\
& \& x_{-i} \gamma \geqslant y_{-i} \delta \quad \Rightarrow \quad v_{-i} \gamma \geqslant w_{-i} \delta
\end{aligned}
$$

for all $x_{-i} \alpha, \ldots, w_{-i} \delta \in E$.

As shown in Wakker (1989b), generalized triple cancellation is equivalent to the requirement that no coordinate should reveal "contradictory (comparisons of) tradeoffs." If $n \geqslant 3$ then in our results generalized triple cancellation can be weakened to the following well-known condition: $\geqslant$ is coordinate independent $(C I)$ on $E$ if $x_{-i} \alpha \geqslant y_{-i} \alpha \Leftrightarrow x_{-i} \beta \geqslant y_{-i} \beta$ for all involved alternatives contained in $E$. That, given reflexivity, CI indeed is implied by generalized triple cancellation can be seen from setting, in the definition of generalized triple cancellation, $\alpha=\beta, \gamma=\delta$, 
$x=y=v$. "Coordinate independence" abbreviates "independence of equal coordinates." On full finite Cartesian products this implies that preferences are independent of all equal/common coordinates, simply because common coordinates can be replaced one by one by other common coordinates, applying CI each time. The latter was called "independence of equal subalternatives" in Wakker (1989b). On general subsets of Cartesian products CI does not have to imply independence of cqual subalternatives because needed "intermediate" alternatives may not be available. As an example, say we have $(1,1,0,0) \succ(1,1,-1,1) \succ(0,0,-1,1) \succ$ $(0,0,0,0)$, with no other alternatives available. CI is trivially satisfied, but the two outer preferences show that preferences are not independent of common subalternatives. On sets of rank-ordered alternatives as considered in this paper, CI nevertheless does imply independence of equal subalternatives. To see this, let $x \geqslant y$, and let $x_{i}=y_{i}$ for all coordinates $i$ in $A \subset\{1, \ldots, n\}$. Let the alternatives that result from $x, y$ by replacing simultaneously all their coordinates $x_{i}=y_{i}, i \in A$ by coordinates $z_{i}$ also be rank-ordered. Then we can also replace the coordinates one by one, as follows. First we replace the $x_{i}=y_{i}$ 's that are preferred to the $z_{i}$ 's, one by one, "from right to left," i.e., first for the largest coordinate $i$ as such, next for the second largest coordinate $i$, etc., until all $x_{i}=y_{i} \geqslant z_{i}$ have been taken care of. Each intermediate alternative was rank-ordered. Next we replace analogously the $x_{i}=y_{i}$ 's that are not preferred to the $z_{i}$ 's, one by one, "from left to right." Each replacement, by $\mathrm{CI}$, does not alter the preference.

\subsection{The Main Results}

One assumption in the main theorems below, not needed in additive representations on full Cartesian products, concerns the monotonicity conditions, together with the related fact that all $n$ product sets are the same set $\mathscr{C}$. This should be no surprise, for without additional structure the very definition of rank-ordering would be impossible.

Assumption 2 [Structural Assumption]. $\mathscr{C}$ is a nonempty set. $\geqslant$ is a binary relation on a set $X \subset \mathscr{C}_{r}^{n}(n \geqslant 2)$ of rank-ordered alternatives, where rank-ordering is with respect to a weak order $\geqslant$ ' on $\mathscr{C}$ "agreeing" with the binary relation $\succcurlyeq$ on $X$ restricted to constant alternatives.

THEOREM 3. Let the structural assumption 2 hold with $X=\mathscr{C}_{r}^{n}$. Let $\geqslant$ satisfy restricted solvability on $X$. The following two statements are equivalent:

(i) There exists an extended monotonic additive representation for $\geqslant$ on $X$ that is real-valued for all nonextreme alternatives.

(ii) The binary relation $\succcurlyeq$ is a weak order satisfying monotonicity, the Archimedean axiom, and generalized triple cancellation.

If $n \geqslant 3$ then generalized triple cancellation in statement (ii) can be weakened to Cl.

Further, the additive representation in (i) is an interval scale on $\mathscr{C}_{r}^{n} \backslash\{$ extreme alternatives $\}$ unless $\geqslant$ on $\mathscr{C}$ has exactly two equivalence classes and $n>3$. To the 
equivalence class of maximal alternatives any value is assigned greater than the values of the other alternatives, to the equivalence class of minimal alternatives any value is assigned less than the values of the other alternatives.

Example 26 will show that, if $\mathscr{C}$ has exactly two equivalence classes, then indeed the additive representation is not an interval scale on $\mathscr{C} \backslash\{$ extreme elements $\}$, the trivial case of $n \leqslant 3$ excepted. (If $n \leqslant 3$ then $\mathscr{C} \backslash\{$ extreme elements\} has no more than two equivalence classes, so that ordinal = cardinal.) Example 25 shows that in statement (i) above indeed non-real-valued representations may occur. The following theorem gives conditions to exclude this.

THEOREM 4. We may add in statement (i) in Theorem 3 that the additive representation is everywhere real-valued if we either:

(a) Add in the theorem the assumption that there are no maximal or minimal outcomes, or

(b) Restrict the domain $X$ of $\geqslant$ to $\mathscr{C}_{r}^{n} \backslash\{$ extreme alternatives $\}$, instead of $\mathscr{C}_{r}^{n}$, or

(c) Strengthen in statement (ii) the Archimedean axiom to the strong Archimedean axiom.

Each of these changes gives again equivalent statements (i) and (ii).

The complicated strong Archimedean axiom instead of the usual one is needed only for the maximal and minimal alternatives. Let us give an alternative condition, a boundedness condition, to guarantee real values at extreme alternatives. The condition does not give a representation result in the strict sense because it is not directly in terms of the observable primitive, the preference relation.

Proposition 5. The extended monotonic additive representation in Theorem 3(i) can be taken real-valued if and only if it is bounded above on $\mathscr{C}_{r}^{n} \backslash\{$ extreme alternatives $\}$ whenever a maximal outcome exists, and bounded below on $\mathscr{C}_{r}^{\prime \prime} \backslash\{$ extreme alternatives $\}$ whenever a minimal outcome exists.

Again, the proportionality condition used in the corollary below is not directly in terms of the preference relation. Conditions that are equivalent to it, and that are directly formulated in terms of the preference relation, have been given in Wakker $(1986,1989 a, 1989 b)$, for the topological approach by "comonotonic cardinal coordinate independence" or "comonotonic noncontradictory tradeoffs" and by Miyamoto (1988), for the algebraic approach when $n=2$, by Axiom 10. Each of these conditions is a small variation on the standard sequence invariance condition in Theorem 6.15(i) of KLST, restricted to rank-ordered subsets. Also Chateauneuf's (1990b) A.5.1 is in this spirit. Nakamura (1990a, 1990b) gave necessary and sufficient conditions for the algebraic approach by variations on the bisymmetry axiom of Pfanzagl (1968), as also studied in Section 6.9 of KLST. Also 
Chew's (1989) "weak commutativity" and Quiggin's (1982) independence are of this nature. Finally, Quiggin (1989, Proposition 5) shows how to characterize the case where additive value functions are identical, thus propertional, by finding a way to adapt the symmetry axiom from full Cartesian products to rank-ordered subsets.

COROLLARY 6. The extended monotonic additive representation in Theorem 3(i) can be taken real-valued everywhere if the additive value functions on $\mathscr{C}_{r}^{n} \backslash\{$ extreme alternatives $\}$ are proportional.

Finally we give a discrete result. The equally spaced case applies if there exist two outcomes $\alpha^{1}>\alpha^{0}$ such that $\alpha^{1}>\beta>\alpha^{0}$ for no outcome $\beta$. In the presence of the other conditions, this will imply that the range of every additive value function can be taken as an interval within the integers, which explains its name. The result below adapts Theorem 3 of Wakker $(1990 \mathrm{c})$ to rank-ordered subsets. It is remarkable that generalized triple cancellation/CI can simply be omitted in the equally spaced case. This was also observed in Doignon \& Falmagne (1974, end of Section II) for the context of difference measurement, as well as in Wakker (1990c), for full Cartesian products. The "weak separability condition" in the latter reference is implied by monotonicity.

COROLlaRY 7. In the equally spaced case the extended monotonic additive representation in Theorem 3(i) can be taken real-valued. The conditions of generalized triple cancellation/CI in statement (ii) can be omitted; they are implied by the other conditions. The range of every additive value function can be taken as an interval within the integers. The additive value functions can be taken identical. All this also holds for Theorem 4(b), i.e., with $X=\mathscr{C}_{r}{ }^{n} \backslash\{$ extreme alternatives $\}$ as domain for $\geqslant$.

\section{Proof of (ii) $\Rightarrow$ (i), ANd the Uniqueness Results, FOR THF. CASE $n \geqslant 3$ WITH CI INSTEAD OF \\ Generalized Triple Cancellation, in Theorem 4(b)}

This section addresses the major part of the proof of the main theorems, i.e., the implication (ii) $\Rightarrow$ (i) in Theorem 4 (b) for the case $n \geqslant 3$ and with Cl. Also we establish the uniqueness results for this case, the case where $\mathscr{C}$ has two equivalence classes excepted; the latter case is dealt with in Example 26.

Let us first point out that, if all outcomes are equivalent, then by monotonicity all alternatives are equivalent, and the result of the theorem is trivial. So we assume throughout this section:

Assumption $8 . \geqslant$ on $\mathscr{C}$ has at least three equivalence classes. The structural assumtion 2 holds with $X=\mathscr{C}_{r}^{n} \backslash\{$ extreme alternatives $\}$ and $n \geqslant 3$. $\geqslant$ is a weak order, it satisfies restricted solvability, the Archimedean axiom, monotonicity, and $\mathrm{CI}$. 
We shall derive statement (i) in Theorem $4(\mathrm{~b})$. A final terminology is the following. We call $\beta$ between $\alpha$ and $\gamma$ if $\alpha \geqslant \beta \geqslant \gamma$ or $\gamma \geqslant \beta \geqslant \alpha$; we say strictly between if the preferences are strict.

\subsection{The Equally Spaced Case; Proof of Corollary 7}

In this subsection we assume we are in the following case, as considered in Corollary 7, given Assumption 8. Let us repeat that, if there is only one equivalence class in $\mathscr{C}$, then everything follows directly from monotonicity; for two equivalence classes see Example 26.

Case 1 . The equally spaced case applies.

So there exist two outcomes $\alpha^{1}>\alpha^{0}$ such that $\alpha^{1}>\beta>\alpha^{0}$ for no outcome $\beta$. This subsection adapts the proof in Wakker $(1990 \mathrm{c})$ to the case of rank-ordered sets.

LEMMA 9. Let $\alpha>\gamma, \sigma>\tau$. Let $w_{-i, j} \alpha, \tau>w_{-i, j} \gamma, \sigma$. Then there exists $\beta$ strictly between $\alpha$ and $\gamma$ such that $w_{-i, j} \beta, \tau \sim w_{-i, j} \gamma, \sigma$.

Proof. Apply restricted solvability to the implied preferences $w_{-i, j} \alpha, \tau>$ $w_{-i, j} \gamma, \sigma>w_{-i, j} \gamma, \tau$. Note that the latter alternative is rank-ordered.

We also have

$$
\exists \sigma \succ \tau: w_{-i, j} \alpha^{1}, \tau \succ w_{-i, j} \alpha^{0}, \sigma
$$

because that would imply, by Lemma 9, existence of $\beta$ strictly between $\alpha^{0}$ and $\alpha^{1}$. Now we construct a kind of standard sequence. We start with the outcomes preferred to $\alpha^{1}$. Set $w:=\left(\alpha^{1}, \ldots, \alpha^{1}\right)$. Suppose we have already constructed $\alpha^{k} \succ \alpha^{0}$ for some $k \geqslant 1$, and there exists $\beta \succ \alpha^{k}$. By (2) we must have $w_{-1, n} \alpha^{k}, \alpha^{1} \preccurlyeq w_{-1, n} \beta, \alpha^{0}$. By Lemma 9 there exists $\alpha^{k+1} \succ \alpha^{k}$ such that $w_{-1, n} \alpha^{k}, \alpha^{1}$ $\sim w_{-1, n} \alpha^{k+1}, \alpha^{0}$. There can be no outcome $\beta$ strictly between $\alpha^{k}$ and $\alpha^{k+1}$ (substitute $\beta$ for $\alpha^{k+1}$ in the last equivalence and apply (2)). We can inductively continue this process. Either it will give an infinite sequence, for which there can be no upper bound by the Archimedean axiom, or the sequence will stop at a maximal $\alpha^{k}$.

Next we consider the outcomes dispreferred to $\alpha^{0}$. Now we set $w:=\left(\alpha^{0}, \ldots, \alpha^{0}\right)$ and we construct analogously $\alpha^{-1}, \ldots$, such that $w_{-1, n} \alpha^{1}, \alpha^{-k} \sim w_{-1, n} \alpha^{0}, \alpha^{-k+1}$ for all $k$. Either this gives an infinite sequence, for which there can be no lower bound by the Archimedean axiom, or the sequence will stop at a minimal $\alpha^{-k^{\prime}}$.

Note that the choice of the $\alpha^{k}$ s is unique only up to equivalence; we could have replaced any $\alpha^{k}$ by any $\beta^{k} \sim \alpha^{k}$. This would affect nothing relevant in our analysis. As in Wakker (1990c), the crucial observation is as follows:

Remark 10. For any $\alpha^{z}$ and $\alpha^{z+1}$ there is no outcome strictly between them. So, loosely speaking, $\alpha^{z+1}$ is the direct follower of $\alpha^{z}$. For any outcome $\beta$ there is an $\alpha^{2} \sim \beta$. 
This shows that the construction of the "quasi-standard sequence" is independent, up to equivalent outcomes, of the particular way of construction.

For any outcome $\beta$ and any $j(j \neq 1$ if $\beta$ is minimal, $j \neq n$ if $\beta$ is maximal) we define $V_{j}(\beta)=z$ with $\alpha^{z} \sim \beta$. It suffices to show that these are additive value functions on the grid alternatives; i.e., the alternatives with all coordinates of the form $\alpha^{z}$.

The following equivalence follows directly for all involved alternatives from Lemma 9 and Remark 10.

$$
w_{-i, j} \alpha^{z}, \alpha^{z^{\prime}+1} \sim w_{-i, j} \alpha^{z+1}, \alpha^{z^{\prime}}
$$

In words, for "tradeoffs of size one" indeed the additive value functions represent equivalences in the right way. Next suppose that for two grid alternatives $x, y$ we have $\sum V_{j}\left(x_{j}\right)=\sum V_{j}\left(y_{j}\right)$. To show that $x, y$ are equivalent, we show that $x$ can be obtained from $y$ by a number of tradeoffs of size 1, i.e., as in Eq. (3). In the present rank-ordered setup this is more complicated than in usual additive conjoint measurement; compare Wakker (1990c, below Eq. (7)) or Wakker (1989b, Step 3.3 in Section III.5). The exact details are somewhat tedious. Our method will be closely related to the "procedure for transforming $x$ into the direction of $y$ " in the proof of Lemma 16; see Figs. 3a and 3b. Say there are $y_{i} \succ x_{i}$ and $x_{j} \succ y_{j}$; if not then the result is trivial. Take the smallest such $i$ and the largest such $j$. If $i>1$, then $x_{i-1} \geqslant y_{i-1} \geqslant y_{i} \succ x_{i}$; if $j<n$, then $x_{j+1} \preccurlyeq y_{j+1} \preccurlyeq y_{j}<x_{j}$. These preferences show that, with say $x_{i}=\alpha^{z}$ and $x_{j}=\alpha^{z^{\prime}+1}, x_{-i, j} \alpha^{z+1}, \alpha^{z^{\prime}}$ is again a rank-ordered alternative. By (3) it is equivalent to $x$. We have carried out a "tradeoff of size 1 into the direction of $y$," without leaving the equivalence class of $x$ and without changing the sum of the additive value functions. The procedure can be continued, and will stop only if the two alternatives have all outcomes equivalent, i.e., identical. Obviously, increasing the sum of the $V_{j}$ 's leads to a higher equivalence class. So the additive function gives an additive representation.

Finally we derive the uniqueness results. They are trivial for the case of one equivalence class of outcomes, and are considered in Example 26 for the case of exactly two equivalence classes of outcomes. So let us assume that there are $\alpha^{1} \succ \alpha^{0} \succ \alpha^{-1}$, with no outcomes strictly between them. First we set $V_{1}\left(\alpha^{1}\right):-1$, and $V_{j}\left(\alpha^{0}\right):=0$ for all $j$, and show that this uniquely determines all $V_{j}$ 's on their domains. We get, by induction with respect to $k, V_{j}\left(\alpha^{-k}\right)=-k$ for all $j>1$ and $k>0$ by setting $x_{1}=\cdots=x_{j}=\alpha^{0}, x_{j+1}=\cdots=x_{n}=\alpha^{-k}$, and by the equivalences $x_{-1, j} \alpha^{1} \alpha^{-k} \sim x_{-1, j} \alpha^{0} \alpha^{-(k-1)}$. Next we get, by induction with respect to $k$, $V_{j}\left(\alpha^{k}\right)=k$ for all $j<n$ and $k>0$ by setting $x_{1}=\cdots=x_{j}=\alpha^{k}, x_{j+1}=\cdots=x_{n}=\alpha^{0}$, and by the equivalences $x_{-j, n} \alpha^{k} \alpha^{-1} \sim x_{-j, n} \alpha^{k-1} \alpha^{0}$. For all nonminimal $\alpha^{-k}(k>0)$ we get, by induction with respect to $k, V_{1}\left(\alpha^{-k}\right)=-k$, by the equivalences $\left(\alpha^{-k}, \alpha^{-k}, \ldots, \alpha^{-k}, \alpha^{-k}\right) \sim\left(\alpha^{-(k-1)}, \alpha^{-k}, \ldots, \alpha^{-k}, \alpha^{-(k+1)}\right)$. Analogously, for all nonmaximal $\alpha^{k}(k>0)$ we get, by induction with respect to $k, V_{n}\left(\alpha^{k}\right)=k$, by the equivalences $\left(\alpha^{k}, \alpha^{k}, \ldots, \alpha^{k}, \alpha^{k}\right) \sim\left(\alpha^{k+1}, \alpha^{k}, \ldots, \alpha^{k}, \alpha^{k-1}\right)$. Note that by the exclusion of extreme alternatives minimal outcomes are not contained in the domain of $V_{1}$, 
and maximal outcomes are not contained in the domain of $V_{n}$. Finally one easily verifies that each other choice $V_{j}\left(\alpha^{0}\right)=\mu_{j}$ for $j=1, \ldots, n$, and $V_{1}\left(\alpha^{1}\right)=\mu_{1}>\mu_{0}$ would uniquely determine an additive representation that is a positive affine transformation of the one established above.

\subsection{The Densely Spaced Case}

Case 1, the equally spaced case, has been taken care of in the previous subsection. From now on we assume we are in the complement of Case 1, i.e.:

Case 2 (The Densely Spaced Case). For all outcomes $\alpha \succ \gamma$ there exists an outcome $\beta$ such that $\alpha>\beta>\gamma$.

Formula (16) below shows that in this case the range of additive representations will be dense. Below we closely follow the line of reasoning in Wakker (1989b, Subsections VI.7.2 and VI.7.3). The following lemma is used in the proof of Lemmas 17 and 21 .

LEMMA 11. Let there be given $x, y, i$ such that $x \succ y, x_{i} \succ x_{i+1}$ if $i<n$, and $x_{i}$ is nonminimal if $i=n$. Then there exists $\alpha$ such that $x_{i} \succ \alpha, \alpha \succ x_{i+1}$ if $i<n$, and $x_{-i} \alpha>y$.

Analogously, suppose $x \prec y, x_{i} \prec x_{i-1}$ if $i>1$, and $x_{i}$ is nonmaximal if $i=1$. Then there exists $\alpha$ such that $x_{i} \prec \alpha, \alpha \prec x_{i-1}$ if $i>1$, and $x_{-i} \alpha \prec y$.

Proof. We prove only the first part. If $i=n$ and $x_{i}$ is nonminimal, define $x_{i+1}$ as any $x_{i+1} \prec x_{i}$. If $x_{-i} x_{i+1} \succ y$, then we set $\alpha:=x_{i+1}$. So say $x_{-i} x_{i+1} \preccurlyeq y$. By restricted solvability there exists $\beta$ between $x_{i}$ and $x_{i+1}$ such that $x_{-i} \beta \sim y$. By monotonicity, $\beta \prec x_{i}$. We can, by the assumption of dense spacedness, take $\beta \prec \alpha \prec x_{i}$. By monotonicity, $x_{-i} \alpha>y$.

We further specify the Case 2 under consideration, i.e., we assume for the time being that we are in Case 2.1, as considered in Theorem 4(a):

Case 2.1 (The Nonmaxmin Densely Spaced Case). There exist no maximal or minimal outcomes.

Case 2.2, the remainder of Case 2, is considered in Subsection 3.6.

\subsection{Additive Representation on Sets $E^{=}$}

Definition 12 [See Fig. 2]. For $z \in \mathscr{C}, E^{z}:=E_{1}^{z} \times \cdots \times E_{n}^{z}$, with $E_{1}^{z}:=\{\alpha \in \mathscr{C}:$ $\left.\alpha \succcurlyeq z_{1}\right\}, E_{n}^{z}:=\left\{\alpha \in \mathscr{C}: z_{n-1} \geqslant \alpha\right\}$, and, for all $1<j<n, E_{j}^{z}:=\left\{\alpha \in \mathscr{C}: z_{j-1} \geqslant\right.$ $\left.\alpha \geqslant z_{j}\right\}$.

Note that $z_{n}$ plays no role in the definition of $E^{z}$, and that coordinates 1 and $n$ are essential since there exist no maximal or minimal outcomes. Coordinates 1 and $n$ are the only essential coordinates if and only if $z_{1} \sim z_{n-1}$, which holds if and only if $E^{z}$ contains a constant alternative; see Fig. 2 and Formula (5) below. 

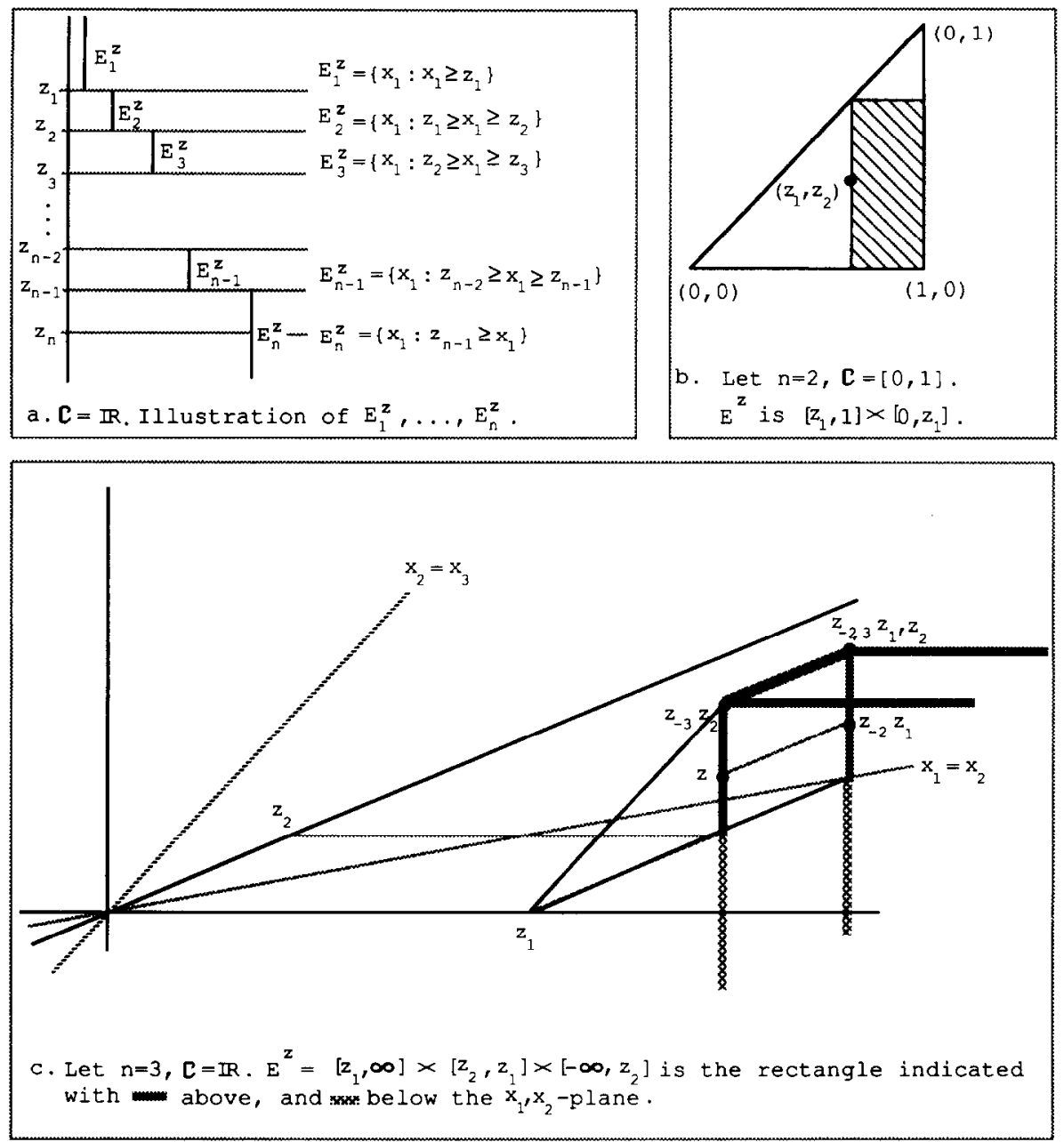

Fig. 2. Illustrations of $E^{z}$. Note that $z_{n}$ plays no role.

LEMMA 13. Let $z_{1}>z_{n-1}$. Then there exists an additive representation $x \mapsto \sum_{j=1}^{n} V_{j}^{x}\left(x_{j}\right)$ of $\geqslant$ on $E^{z}$ that is an interval scale.

Proof. By Theorem 6.13 in KLST the results (4), (5), and (6) below together imply the lemma. It is obvious that:

$E^{z}$ is a Cartesian product, $\geqslant$ is a weak order on $E^{z}, \geqslant$ is CI on $E^{z}$, and $\succcurlyeq$ satisfies the Archimedean axiom on $E^{z}$.

Next we show, using monotonicity:

$\geqslant$ has three or more essential coordinates on $E^{z}$. 
Since $z_{1}$ is not maximal, there is $x_{1}>z_{1}$; hence $\geqslant$ has the first coordinate essential on $E^{z}$. Analogously nonminimalness of $z_{n-1}$ implies essentiality of the $n$th coordinate on $E^{z}$, and $z_{1}>z_{n-1}$ implies that besides coordinates 1 and $n$ one more coordinate must be essential.

Finally we show:

$$
\geqslant \text { satisfies restricted solvability. }
$$

Suppose, for some $x_{-i} \alpha, y, x_{-i} \gamma \in E^{z}, x_{-i} \alpha \succ y>x_{-i} \gamma$. By restricted solvability of $\succcurlyeq$ on $X$, there exists $\beta$ such that $x_{-i} \beta \sim y$. It staightforwardly follows from monotonicity that $\alpha>\beta>\gamma$. From this follows that $\beta \in E_{i}^{z}$, thus $x_{-i} \beta \in E^{z}$. Restricted solvability of $\geqslant$ on $E^{z}$ holds.

\subsection{Fitting the Functions $V_{j}^{z}$ Together, Giving a Global Additive Function which Is} Locally Representing

The "local" additive representations through the functions $V_{j}^{z}$ are interval scales, so a scale and location constant can still be chosen freely for each of them. In this subsection we prove the following lemma, showing that these scales and locations can be chosen so that all $V_{j}^{z}$ 's coincide on common domains. Loosely speaking, the domains of these functions have sufficient overlap. Below we do not yet have a "global" representation, so we cannot call the $V_{j}^{\prime}$ 's additive value functions.

Lemma 14. There exists an additive interval scale $V: x \mapsto \sum_{j=1}^{n} V_{j}\left(x_{j}\right)$ on $X$ which represents $\geqslant$ on every $E^{z}$ with three or more essential coordinates; every $V_{j}$ represents $\geqslant$ on its domain $\mathscr{C}$.

Proof. We fix outcomes $\beta^{1}>\beta^{0}$.

Stage 1. Choice of "reference points."

We fix some $r^{0}$ and $r^{1}$ such that, obviously, $r_{1}^{0} \succ r_{n-1}^{0}, r_{1}^{1} \succ r_{n-1}^{1}$; further $E_{1}^{r^{\prime}}$ contains both $\beta^{0}$ and $\beta^{1}$, and $E_{n}^{r^{0}}$ contains $\beta^{0}$; note that by $r_{1}^{1}>r_{n-1}^{1}, \beta^{0}$ cannot be contained in $E_{n}^{r^{1}}$. We choose locations so that $V_{n}^{r^{0}}\left(\beta^{0}\right)=0$ and $V_{1}^{r}\left(\beta^{0}\right)=0$. Further we choose scale so that $V_{1}^{r^{1}}\left(\beta^{1}\right)=1$.

Stage 2. Fitting together the $V_{1}^{z}$ 's and $V_{n}^{z}$ 's.

For all $z \in X$ with $z_{1}>z_{n-1}$, and for $r=r^{0}$ as well as $r=r^{1}$, the following paragraph applies:

By CI, and independence of equal subalternatives as implied by $\mathrm{CI}$ (see end of Subsection 2.3), $\left(V_{1}^{r}, V_{n}^{r}\right)$ and $\left(V_{1}^{z}, V_{n}^{z}\right)$ are additive value functions for the same $\succcurlyeq_{\{1, n\}}$ on $\left(E_{1}^{r} \cap E_{1}^{z}\right) \times\left(E_{n}^{r} \cap E_{n}^{z}\right)$. Note that both coordinates 1 and $n$ are essential on $\left(E_{1}^{r} \cap E_{1}^{z}\right) \times\left(E_{n}^{r} \cap E_{n}^{z}\right)$ with respect to $\succcurlyeq_{\{1, n\}}$. This will be used several times, without explicit mentioning, in the sequel. This $\succcurlyeq_{\{1, n\}}$ satisfies the Archimedean axiom and restricted solvability, as follows from the same conditions for $\geqslant$ on $X$ and, mainly, $\mathrm{CI}$ and monotonicity. Hence we can use the standard uniqueness results from two-dimensional additive representations (see KLST, Theorem 6.2). 
We first choose locations of all $V_{1}^{z}$ 's, and scales of all $\left(V_{1}^{z}, V_{n}^{z}\right)$ 's (thus scales of all $\left.\left(V_{j}^{z}\right)_{j=1}^{n}\right)$ so that $V_{1}^{r^{\prime}}=V_{1}^{z}$ on common domain. This includes $z=r^{0}$. Next we choose locations for all $V_{n}^{z}$ so that they coincide with $V_{n}^{r^{0}}$ on common domain; this includes $z=r^{1}$.

We shall show, even stronger,

$$
\begin{aligned}
& V_{1}^{s}=V_{1}^{t} \text { and } V_{n}^{s}=V_{n}^{t} \text { on common domain for all } s, t \text { for which } \\
& s_{1} \succ s_{n-1} \text { and } t_{1} \succ t_{n-1} .
\end{aligned}
$$

This follows since, on $\left(E_{1}^{s} \cap E_{1}^{t}\right) \times\left(E_{n}^{s} \cap E_{n}^{t}\right),\left(V_{1}^{s}, V_{n}^{s}\right)$ and $\left(V_{1}^{t}, V_{n}^{t}\right)$ are additive value functions for the same $\geqslant_{\{1, n\}}$, so that they can differ only with respect to their locations, and a common scale. However, $V_{1}^{s}$ and $V_{1}^{t}$ coincide (with $V_{1}^{r^{1}}$ ) on $E_{1}^{s} \cap E_{1}^{t} \cap E_{1}^{r^{1}}$, and $V_{n}^{s}$ and $V_{n}^{t}$ coincide (with $V_{n}^{r^{0}}$ ) on $E_{n}^{s} \cap E_{n}^{t} \cap E_{n}^{r^{0}}$; hence they coincide on common domains.

The rest of this subsection closely follows Wakker (1989b, p. 124).

Stage 3. Intermediate observation.

For all $s, t$ with $s_{1} \succ s_{n-1}, t_{1} \succ t_{n-1}$ and all $j, V_{j}^{s}$ and $V_{j}^{t}$ now have the same scale, and differ only with respect to their location, as we shall show:

For all $s, t \in X$ with $s_{1}>s_{n-1}$ and $t_{1}>t_{n-1}$ and $1 \leqslant j \leqslant n$ there exist constants $\tau_{j}(s, t)$ such that on $E_{j}^{s} \cap E_{j}^{t}, V_{j}^{s}=\tau_{j}(s, t)+V_{j}^{t}$.

For $j=1$ or $j=n$, by $(7), \tau_{j}(s, t)=0$. So let $1<j<n$. Then $\left(V_{1}^{s}, V_{j}^{s}, V_{n}^{s}\right)$ and $\left(V_{1}^{t}, V_{j}^{t}, V_{n}^{t}\right)$ are additive value functions for the same $\geqslant_{\{1, j, n\}}$ on $\left(E_{1}^{s} \cap E_{1}^{t}\right) \times$ $\left(E_{j}^{s} \cap E_{j}^{t}\right) \times\left(E_{n}^{s} \cap E_{n}^{t}\right)$. So they can differ only by location and common scale. However, $V_{1}^{s}$ and $V_{1}^{t}$, and $V_{n}^{s}$ and $V_{n}^{t}$, coincide on their common domain (which contains more than one equivalence class). The common scales must be the same.

Stage 4. Choice of location for all $V_{j}^{z}$ s $(1<j<n)$, having $\beta^{0}$ in their domain. have

Of course for all $V_{j}^{z \prime}$ s as above we choose location such that $V_{j}^{z}\left(\beta^{0}\right)=0$. Now we

If $V_{j}^{s}$ and $V_{j}^{t}$ have $\beta^{\circ}$ in their domain then they coincide on common domain.

This follows directly from (8).

Stage 5. Choice of location for remaining $V_{j}^{z}$ 's.

Now let $z \in X$ with $z_{1}>z_{n-1}$ and $j$ be such that $1<j<n, \beta^{0} \notin E_{j}^{z}$. Consider the "boundary element" of $E_{j}^{z}$ that is "closest" to $\beta^{0}$ (i.e., $z_{j-1}$ if $z_{j-1} \prec \beta^{0}, z_{j}$ if $z_{j} \succ \beta^{0}$ ). Obviously that boundary element is determined up to $\sim$-equivalence. Let $V_{j}^{z}$ coincide at this boundary point, thus by (8) on entire common domain, with one, thus every, $V_{j}^{s}$ with $s$ such that $s_{1}>s_{n-1}, \beta^{0} \in E_{i}^{s}$, and $E_{i}^{s}$ intersects $E_{i}^{z}$, thus intersects it in the boundary element. 
Now we have

For all $s, t \in X, 1 \leqslant j \leqslant n, V_{j}^{s}$ and $V_{j}^{\prime}$ coincide on common domain.

The only case for which this has not yet been established is the case where $1<j<n$ and $\beta^{0}$ is neither in the domain of $V_{j}^{s}$, nor in that of $V_{j}^{t}$. We can always find $V_{j}^{z}$ that has $\beta^{0}$ in its domain, and contains the entire domains of $V_{j}^{s}$ and $V_{j}^{t}$. Both $V_{j}^{s}$ and $V_{j}^{t}$ must coincide with $V_{j}^{z}$, thus with each other on common domain.

Because of the absence of maximal and minimal outcomes, for every $j$ the union of the domains $E_{j}^{z}$ is the entire $\mathscr{C}$; by $(10), V_{j}^{z}(\alpha)$, whenever defined, is independent of the superindex $z$. Hence we can leave out the superindexes to get $V_{j}: \mathscr{C} \rightarrow \mathbb{R}$ for $j=1, \ldots, n$.

OBSERVATION 15. Note that in Stage 1 the choices of locations were fully arbitrary, as was the choice of the scale through $V_{1}^{r^{1}}\left(\beta^{1}\right)$, as long as $V_{1}^{r^{1}}\left(\beta^{1}\right)>V_{1}^{r^{1}}\left(\beta^{0}\right)$. $A$ rereading of the proof shows that given these choices the additive value functions are fully determined, and that they are interval scales.

Finally we show that every $V$, represents $\succcurlyeq$ on $\mathscr{C}$. Let $\alpha, \beta \in \mathscr{C}$. We can find an $E^{z}$ with three or more essential coordinates and with $\alpha, \beta \in E_{j}^{z}$. By monotonicity $\alpha \geqslant \beta \Leftrightarrow z_{-j} \alpha \geqslant z_{-j} \beta$. On $E^{z}, V_{1}, \ldots, V_{n}$ are additive value functions, hence $z_{-j} \alpha \geqslant$ $z_{-j} \beta \Leftrightarrow V_{j}(\alpha) \geqslant V_{j}(\beta)$.

\subsection{The Global Additive Function is Not Only Locally, but Also Globally, Repre- senting}

It has been overlooked several times in the literature that at this stage it may not yet be concluded that $\left(V_{j}\right)_{j=1}^{n}$ are global additive value functions, i.e., additive value functions on the entire set $X$. Only for alternatives $x, y$ from a same $E^{z}$ the equivalence $\left[x \geqslant y \Leftrightarrow \sum V_{j}\left(x_{j}\right) \geqslant \sum V_{j}\left(y_{j}\right)\right]$ has been established so far. Wakker (1989b, Remark III.7.8) shows that for general subsets of Cartesian products, even if these subsets are connected and "full-dimensional," the step from local to global additive representation may go wrong. It may for general subsets of Cartesian products happen that two "remote" elements $x, y$ of $X$ are equivalent, but their $V$-value is different. In our present context global representation will nevertheless be demonstrated. The $E$ 's turn out to have "sufficient overlap." The general idea is to construct for, say, equivalent and remote alternatives $x, y$, a sequence of alternatives $x^{0}, \ldots, x^{m}$ on the equivalence class of $x, y$ so that $x^{0}=x, x^{m}=y$, and every subsequent pair $x^{j}, x^{j+1}$ lies within one common $E^{z}$. The latter will guarantee that $V$ assigns the same value to each pair $x^{j}, x^{j+1}$, thus to $x^{0}=x$ and $x^{m}=y$. The actual construction of the sequence is a tedious process that can be carried out in several ways. Details are provided in the proof below.

Lemma 16. $V$ is representing on the set $\left\{x \in X: x_{1}>x_{n-1}\right\}$.

Proof. Let $x \geqslant y$ be in $\left\{v \in X: v_{1} \succ v_{n-1}\right\}$. We start the proof by describing a procedure to transform $x$ into an equivalent $x^{\prime}$ that has the same $V$-value as $x$ and 
that is "closer" to $y$ in the sense that $x^{\prime}$ has at least one more coordinate identical to $y$ than $x$. The procedure can be applied whenever there is a coordinate $i$ for which $x_{i} \prec y_{i}$. A special case of the procedure can be recognized in the proof of Lemma VI.7.10 in Wakker (1989b). A first reason for the generalization needed here is that in the present nontopological setup there does not have to exist a "certaintyequivalent" for each alternative; for instance in Example III.6.8 of Wakker (1989b), where one can also consider the rank-ordered subset, there does not exist a certainty equivalent for $(\sqrt{2}, 0)$. A second reason is that the constant alternatives are not contained in an $E^{z}$ with three or more essential coordinates, and contrary to Wakker (1989b) these are the only $E^{z \prime}$ s considered here.

\section{The Procedure for Transforming $x$ into the Direction of $y$}

Beginning of procedure. See Fig. 3. Suppose $x_{i} \prec y_{i}$. Let us take the smallest such $i$ (so the most preferred of those $x_{i}^{\prime}$ 's). Note that either $i$ is 1 , or $x_{i} \prec y_{i} \preccurlyeq$ $y_{i-1} \preccurlyeq x_{i-1}$ and $y_{k} \preccurlyeq x_{k}$ for all $k<i$. By monotonicity there must be $x_{j} \succ y_{j}$. Let us take the largest such $j$. Either $j$ is $n$, or $x_{j}>y_{j} \geqslant y_{j+1} \geqslant x_{j+1}$ and $y_{k} \geqslant x_{k}$ for all $k>j$. We shall increase $x_{i}$ and decrease $x_{j}$, thus kind of move into the direction of $y$, in a way to stay within the equivalence class of $x$. Note that $x_{-i, j} y_{i}, y_{j} \in X$. If $x_{-i, j} y_{i}, y_{j} \geqslant x$ then by restricted solvability we can find $x_{i}^{\prime}$ between $x_{i}$ and $y_{i}$ such that $x^{\prime}=\left(x_{1}^{\prime}, \ldots, x_{n}^{\prime}\right):=x_{-i, j} x_{i}^{\prime}, y_{j} \sim x$.

If $x_{-i, j} y_{i}, y_{j} \prec y$ then by restricted solvability we can find $x_{j}^{\prime}$ between $x_{j}$ and $y_{j}$ such that $x^{\prime}=\left(x_{1}^{\prime}, \ldots, x_{n}^{\prime}\right):=x_{-i, j} y_{i}, x_{j}^{\prime} \sim x$.

In any case we have: both $x$ and $x^{\prime}$ are in $E^{z}$ for $z:=x_{-i, j} x_{i}, y_{j}$, further, as we shall show below, this $z$ has $z_{1}>z_{n-1}$. Thus $x$ and $x^{\prime}$ have the same $V$-value.

Let us next show that $x_{1}^{\prime} \succ x_{n}^{\prime} \quad 1$ and, for $z$ as above, $z_{1}>z_{n} \quad{ }_{1}$. First we consider some, not mutually exclusive, cases, and derive observations implying the desired preferences. Then we show that these cases are exhaustive. The notation $\left\{a_{j}, b_{j}\right\} \geqslant c_{j}$ means that both $a_{j} \succcurlyeq c_{j}$ and $b_{j} \succcurlyeq c_{j}$.

Case 1. $\{i, j\} \cap\{1, n-1\}=\varnothing$. Then $\left(x_{1}^{\prime}, x_{n-1}^{\prime}\right)=\left(x_{1}, x_{n-1}\right)=\left(z_{1}, z_{n-1}\right)$.

Case 2. $i<j$ and $i \neq n-1$. Then $\left\{x_{1}^{\prime}, z_{1}\right\} \geqslant x_{1}>x_{n-1} \geqslant\left\{x_{n-1}^{\prime}, z_{n-1}\right\}$.

Case 3. $j=1$ or $i=n-1$. Then $\left\{x_{1}^{\prime}, z_{1}\right\} \geqslant y_{1} \succ y_{n-1} \geqslant\left\{x_{n-1}^{\prime}, z_{n-1}\right\}$.

Case 4. $j=n-1$ and $i=n$. Then $x_{1}^{\prime}=x_{1} \succ x_{n-1} \succ x_{n-1}^{\prime}$ and $z_{1}=x_{1} \succ x_{n-1} \succ$ $y_{n-1}=z_{n-1}$.

Let us show that this covers all possibilities. Suppose we are not in Case 1. Then $i=1$ or $i=n-1$ or $j=1$ or $j=n-1 . i=1$ has been dealt with in Case 2 above, $i=n-1$ or $j=1$ in Case 3, $j=n-1$ either in Case 2 or in Case 4. End of procedure.

Since both $x$ and $x^{\prime}$ in the above procedure are contained in one same $E^{z}$, their equivalence implies equality of their $V$-value. The procedure can be continued as long as there is a coordinate $i$ for which $x_{i} \prec y_{i}$. At each step of the procedure, at least one more coordinate becomes identical to one of $y$, thus after at most $n$ times 

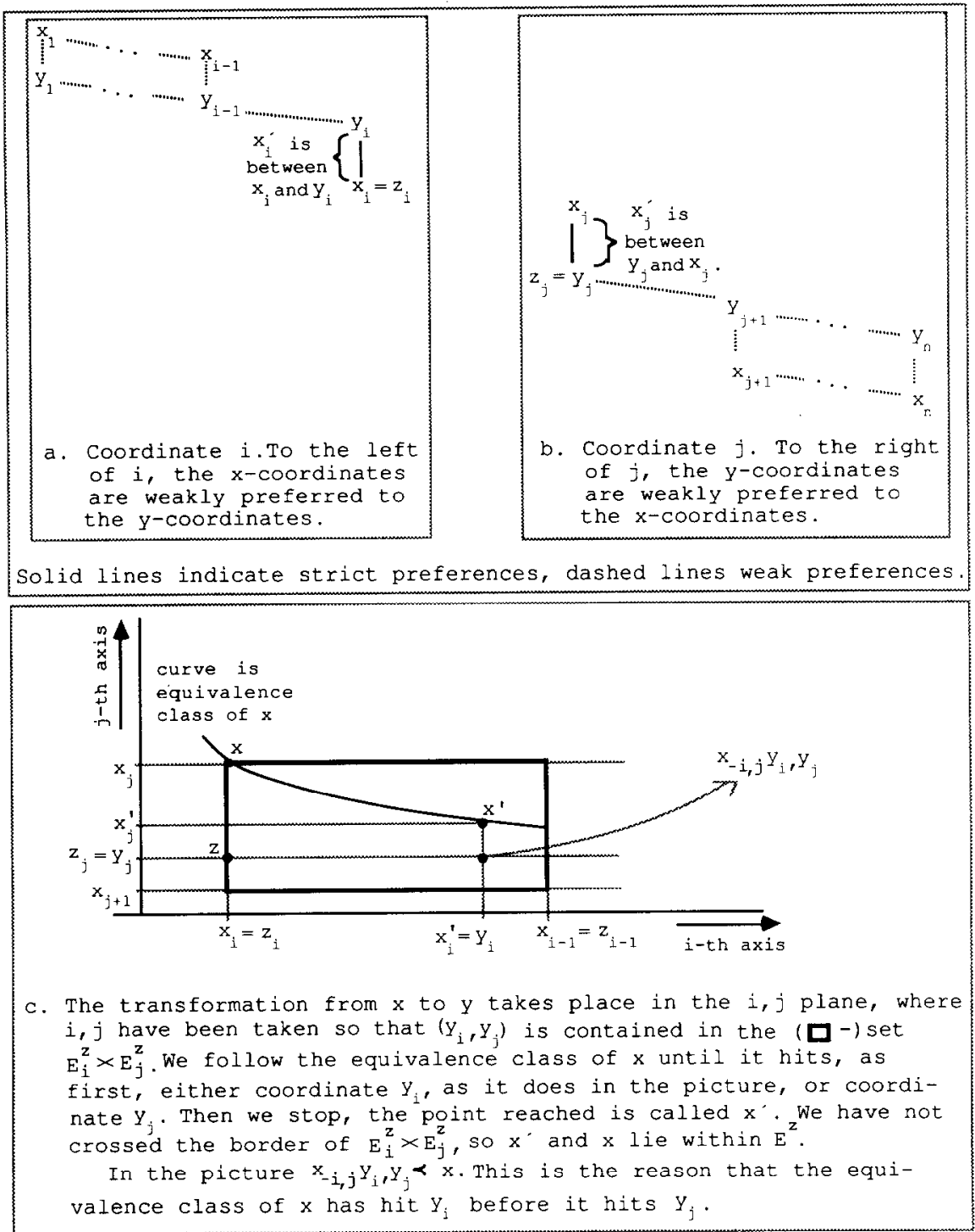

FIG. 3. The procedure for transforming $x$ into the direction of $y$.

the procedure must stop. Then all coordinates of the resulting alternative must be weakly preferred to those of $y$. If $x$ is equivalent to $y$ then so must the obtained alternative be, so by monotonicity all its coordinates must be equivalent to those of $y$. Since by Lemma 14 the $V_{j}$ 's represent $\geqslant$ on $\mathscr{C}, V_{j}\left(x_{j}\right)=V_{j}\left(y_{j}\right)$ follows for all $j$. Hence $V(x)=V(y)$. If $x$ is strictly preferred to $y$ then so is the obtained alternative, so by monotonicity it must have at least one coordinate strictly preferred to 
$y$, and $V(x)>V(y)$ follows. So $x>y \Rightarrow V(x)>V(y), x \sim y \Rightarrow V(x)=V(y)$. This implies that $V$ is representing.

Lemma $17 . \quad V$ is representing on the entire $X$.

Proof. We shall show $x>y \Leftrightarrow V(x)>V(y)$.

First suppose $x>y$. To show is that $V(x)>V(y)$. By Lemma 11 we can "decrease" coordinates $n$ and $n-1$ of $x$ somewhat, and "increase" coordinate 1 of $y$ somewhat, under preservation of the strict preference. The desired $V$-inequality then follows from Lemmas 16 and 14 . We have established

$$
x>y \Rightarrow V(x)>V(y) \text {. }
$$

Since $\geqslant$ is a weak order we now also have

$$
V(x) \geqslant V(y) \Rightarrow x \geqslant y, \quad V(x)>V(y) \Rightarrow x \geqslant y, \quad V(x)=V(y) \Rightarrow x \sim y .
$$

For $V$ to be representing, we must exclude the case $[x \sim y$ and $V(x)>V(y)]$. Suppose $V(x)>V(y)$. We show that $x>y$. There must be a coordinate $j$ with $V_{j}\left(x_{j}\right)>V_{j}\left(y_{j}\right)$. We take the largest of such $j$ s. So either $j$ is $n$, or

$$
x_{j} \succ y_{j} \succcurlyeq y_{j+1} \succcurlyeq x_{j+1} \text {. }
$$

(Compare Fig. 3b.) It suffices to find an $x_{j}^{\prime}$ strictly between $x_{j}$ and $y_{j}$, "close enough" to $x_{j}$ to give $V\left(x_{-j} x_{j}^{\prime}\right)>V(y)$. (Note that, if $j<n$, then by (13) $x_{j}^{\prime}>x_{j+1}$ so that indeed $x_{-j} x_{j}^{\prime} \in X$.) Then by (12), $x_{-j} x_{j}^{\prime} \geqslant y$, and by monotonicity $x>y$ follows. The remainder of this proof constructs $x_{j}^{\prime}$. The construction is technical.

Say $j<n$. The case $j>1$ can (and the case $j=n$ must) be treated in a different but analogous manner. One can then construct a "fine measure stick" $v_{1}>w_{1}$ on coordinate 1 with $w_{1} \geqslant\left\{x_{1}, y_{1}\right\}$, to be used analogously to the "fine measure stick" $v_{n}>w_{n}$ on coordinate $n$ as constructed below.

There exist $\left\{x_{n}, y_{n}\right\} \geqslant \alpha^{0}>\gamma^{0}$. We will inductively define $\alpha^{k}>\gamma^{k}$ such that

$$
0<V_{n}\left(\alpha^{k+1}\right)-V_{n}\left(\gamma^{k+1}\right) \leqslant\left[V_{n}\left(\alpha^{k}\right)-V_{n}\left(\gamma^{k}\right)\right] / 2 .
$$

For every $\alpha^{k} \succ \gamma^{k}$ there exists $\beta$ strictly between $\alpha^{k}$, $\gamma^{k}$. If $V_{n}\left(\alpha^{k}\right)-V_{n}(\beta) \geqslant$ $V_{n}(\beta)-V_{n}\left(\gamma^{k}\right)$ then we take $\alpha^{k+1}:=\beta, \gamma^{k+1}:=\gamma^{k}$. In the other case we take $\alpha^{k+1}:=\alpha^{k}, \gamma^{k+1}:=\beta$.

We take $k$ so large that, with $v_{n}:=\alpha^{k}, w_{n}:=\beta^{k}$,

$$
V(x)-V(y)>V_{n}\left(v_{n}\right)-V_{n}\left(w_{n}\right) .
$$

If $x_{-j} y_{j} \geqslant y$ (note that, because of (13), $x_{-j} y_{j}$ is contained in $X$ ) then by monotonicity $x>y$ follows immediately. So suppose $y>x_{-i} y_{i}$. This by (11) 
implies $V(y)>V\left(x_{\ldots,} y_{i}\right)$, which implies the first inequality below. The second is (14).

$$
\begin{aligned}
V_{j}\left(x_{j}\right)-V_{j}\left(y_{j}\right) & =V(x)-V(y)+V(y)-V\left(x_{-j} y_{j}\right) \\
& >V(x)-V(y)>V_{n}\left(v_{n}\right)-V_{n}\left(w_{n}\right)
\end{aligned}
$$

It follows that $x_{-j, n} x_{j}, v_{n} \succ x_{-j, n} x_{j}, w_{n} \geqslant x_{-j, n} y_{j}, v_{n}$, the latter preference by (15) and (12). By restricted solvability there must exist an $\alpha$ such that $x_{-j, n} \alpha, v_{n} \sim$ $x_{-j, n} x_{j}, w_{n}$. By monotonicity $\alpha \prec x_{j}$. In the present Case 2 there exists $x_{j}^{\prime}$ strictly between $x_{j}$ and $\alpha$, by monotonicity $x_{-j, n} x_{j}^{\prime}, v_{n} \succ x_{-j, n} x_{j}, w_{n}$. By (11) we get $V_{n}\left(v_{n}\right)-V_{n}\left(w_{n}\right)>V_{j}\left(x_{j}\right)-V_{j}\left(x_{j}^{\prime}\right)$. This and (14) implies the inequality in $V^{\prime}(x)-V(y)>V_{j}\left(x_{j}\right)-V_{j}\left(x_{j}^{\prime}\right)=V(x)-V\left(x_{-j} x_{j}^{\prime}\right)$. Subtracting right-hand side from left-hand side gives $V\left(x_{-j} x_{j}^{\prime}\right)-V(y)>0$.

The following, easily derivable from the above proof, is used below. It is an analogue of Lemma 11.

The range of each $V_{j}$ is dense within its convex hull.

To wit, let $j<n$. As above, $j>1$ is analogous. Let $V_{j}\left(x_{j}\right)>V_{j}\left(y_{j}\right)$. Analogously to the above proof, we can construct $v_{n}, w_{n}$ with $V_{n}\left(v_{n}\right)-V_{n}\left(w_{n}\right)>0$ arbitrarily small. Next we can construct a "standard-sequence" $y_{j}^{0}, y_{j}^{1}, y_{j}^{2}, \ldots$, with first element $y_{j}^{0}:=y_{j}$ such that $V_{j}\left(y_{j}^{k+1}\right)-V_{j}\left(y_{j}^{k}\right)=V_{n}\left(v_{n}\right)-V_{n}\left(w_{n}\right)$ for all $k$. By the Archimedean axiom the part of the standard sequence not preferred to $x_{j}$ is finite, for the last such $y_{j}^{k}$ we have $V_{j}\left(y_{j}^{k}\right)-V_{j}\left(x_{j}\right)<V_{n}\left(v_{n}\right)-V_{n}\left(w_{n}\right)$. Since $V_{n}\left(v_{n}\right)-V_{n}\left(w_{n}\right)>0$ can be taken arbitrarily small, (16) follows.

\subsection{Maximal and/or Minimal Outcomes}

Now we turn to the complement within Case 2 of Case 2.1:

Case 2.2 (The Maxmin Densely Spaced Case). There exist maximal or minimal outcomes.

$\mathscr{C}^{*}:=\{\alpha \in \mathscr{C}: \alpha$ is neither maximal nor minimal $\}$, and $\left(\mathscr{C}^{*}\right)_{r}^{n}:=\left\{x \in X: x_{j} \in \mathscr{C} *\right.$ for all $j\}$. By Assumption $8, \mathscr{C}$ has more than one equivalence class. So $\mathscr{C}$ and $\mathscr{C} *$ have infinitely many equivalence classes in the present Case 2. Also in Case 2 every nonmaximal outcome is dominated by another nonmaximal outcome, and every nonminimal outcome dominates another nonminimal outcome. We summarize:

Lemma 18. $\mathscr{C}^{*}$ has infinitely many equivalence classes. There are no outcomes in $\mathscr{C}^{*}$ that are maximal or minimal in $\mathscr{C}^{*}$.

Next we show that, on $\left(\mathscr{C}^{*}\right)_{r}^{n}$, (i) in Theorem $4(\mathrm{~b})$ is satisfied.

LEMMA 19. There exist monotonic additive value functions $\left(V_{j}^{*}\right)_{j=1}^{n}$ for $\geqslant$ on $\left(\mathscr{C}^{*}\right)_{,}^{n}$. 
Proof. On $\left(\mathscr{C}^{*}\right)_{r}^{n}, \geqslant$ satisfies:

- $\geqslant$ is a weak order.

- $\geqslant$ satisfies monotonicity.

- $\geqslant$ satisfies the Archimedean axiom.

- $\geqslant$ satisfies restricted solvability. (By monotonicity the "solving $\beta$ " in the definition of restricted solvability must be nonmaximal and nonminimal.)

- $\geqslant$ satisfies CI.

- $\mathscr{C}^{*}$ itself has no maximal or minimal outcomes.

Thus on $\left(\mathscr{C}^{*}\right)_{r}^{n}$ all conditions of Theorem $4(\mathrm{~b})$ are satisfied, as well as the assumption of absence of maximal/minimal outcomes. The result of Case 2.1, the nonmaxmin densely spaced case, applies to $\left(\mathscr{C}^{*}\right)_{r}^{n}$.

We plan to define, for each $j<n$ and each maximal $\mu, V_{j}(\mu):=\sup \left(V_{J}^{*}\left(\mathscr{C}^{*}\right)\right)$, and for each $j>1$ and minimal $v, V_{j}(v):=\inf \left(V_{j}^{*}\left(\mathscr{C}^{*}\right)\right)$. As a preparation we derive the following result.

LEMMA 20. If there exists a maximal outcome then for every $j<n, V_{j}^{*}\left(\mathscr{C}^{*}\right)$ is bounded above. If there exists a minimal outcome then for every $j>1, V_{j}^{*}\left(\mathscr{C}^{*}\right)$ is bounded below.

Proof. Say $\mu$ is maximal (minimal $v$ is analogous). Let $j<n$. Take any $\mu \succ v_{n} \succ w_{n} \in \mathscr{C}^{*}$. Set $x_{i}=\mu$ for all $1 \leqslant i<j, x_{i}=v_{n}$ for all remaining $i$. If $x_{-j, n} \mu, w_{n} \preccurlyeq x_{-j, n} v_{n}, v_{n}$ then $x_{-j, n} \beta, w_{n} \preccurlyeq x_{-j, n} v_{n}, v_{n}$ for any $\beta \in \mathscr{C}^{*}$; substituting additive value functions gives an upper bound for $V_{j}^{*}\left(\mathscr{C}^{*}\right)$.

If $x_{-j, n} v_{n}, v_{n} \prec x_{-j, n} \mu, w_{n} \prec x_{-j, n} \mu, v_{n}$ then by restricted solvability we find $\mu^{\prime}$ such that $x_{-j, n} \mu^{\prime}, v_{n} \sim x_{-j, n} \mu, w_{n}$. This implies $x_{-j, n} \mu^{\prime}, v_{n} \geqslant x_{-j, n} \beta, w_{n}$ for all $\beta \in \mathscr{C}^{*}$, resulting again in an upper bound for $V_{j}^{*}\left(\mathscr{C}^{*}\right)$.

If $\mu \in \mathscr{C}$ is maximal, then for all $j<n$ we define $V_{j}(\mu):=\sup \left(V_{j}^{*}\left(\mathscr{C}^{*}\right)\right)$. If $v \in \mathscr{C}$ is minimal, then for all $j>1$ we define $V_{j}(v):-\inf \left(V_{j}^{*}\left(\mathscr{C}^{*}\right)\right)$. If $\gamma \in \mathscr{C}^{*}$, then $V_{j}(\gamma):=V_{j}^{*}(\gamma)$. As we saw above, $V_{j}(\alpha) \in \mathbb{R}$ whenever it is defined.

Lemma 21. $V$ is an additive representation on $X$.

Proof. We show

$$
x>y \Leftrightarrow V(x)>V(y) .
$$

First suppose $x \succ y$. Let $x_{1}, \ldots, x_{j}$ be maximal, $x_{j+1}$ is not maximal. By Lemma 11 we can find $x_{j}^{\prime} \prec x_{j}$ such that $x_{-j} x_{j}^{\prime} \in X, x_{-j} x_{j}^{\prime} \succ y$. Next we can analogously substitute $x_{j-1}^{\prime} \prec x_{j-1}, \ldots, x_{1}^{\prime} \prec x_{1}$, to end up with $x^{\prime}$ such that still $x^{\prime} \succ y$. It suffices to prove $V\left(x^{\prime}\right)>V(y)$. Analogously we can replace minimal coordinates of $y$ by nonminimal coordinates under preservation of the preference, and it suffices to prove $V\left(x^{\prime}\right)>V\left(y^{\prime}\right)$. We omit accents, and simply assume that $x$ has no maximal 
coordinates, and $y$ no minimal coordinates. $x$ must have a nonminimal coordinate $x_{i}$; let us take the maximal $i$ as such. By Lemma 11 we can find nonminimal $x_{i}^{\prime} \prec x_{i}$ such that still $x^{\prime}:=x_{-i} x_{i}^{\prime} \succ y$. It suffices to prove $V\left(x^{\prime}\right) \geqslant V(y)$. Note that if we replace the minimal coordinates of $x^{\prime}$ by nonminimal ones, and the maximal coordinates of $y$ by nonmaximal ones, then by monotonicity the preference between the two is not affected, and by Lemma 19 the $V$-value of the first is strictly greater than the $V$-value of the second. Since, by the definition of the $V_{j}$ s at minimal outcomes, $V\left(x^{\prime}\right)$ is the infimum of the associated $V$-values, and by the definition of the $V_{j}^{\prime}$ 's at maximal outcomes, $V(y)$ the supremum of the associated $V$-values, $V\left(x^{\prime}\right) \geqslant V(y)$ follows. Obviously (17) also implies

$$
V(x) \geqslant V(y) \Rightarrow x \geqslant y \text {. }
$$

Next suppose $V(x)>V(y) . x$ cannot have all of its coordinates minimal, so say $j$ is the largest coordinate so that $x_{j}$ is not minimal. By (16) we can find a nonminimal $x_{j}^{\prime} \prec x_{j}$ such that still $V\left(x_{-j} x_{j}^{\prime}\right)>V(y)$. By (18), $x_{-j} x_{j}^{\prime} \geqslant y$. By monotonicity, $x>y$.

The following observation prepares for the uniqueness result of Theorem $4(\mathrm{~b})$.

OBSERVATION 22. Let $V$ be an additive representation for $\geqslant$ on $X$. If $\mu$ is a maximal outcome, then for each $j<n, V_{j}(\mu)=\sup \left(V_{j}\left(\mathscr{C}^{*}\right)\right)$. If $v$ is a minimal outcome, then for each $j>1, V_{j}(v)=\inf \left(V_{j}\left(\mathscr{C}^{*}\right)\right)$.

Proof. Let $\mu$ be a maximal outcome, $j<n$. At the end of the proof we construct an equivalence $x_{-j . n} \mu, w_{n} \sim x_{-j, n} \mu^{\prime}, v_{n}$ with nonextreme $\mu^{\prime}, v_{n}, w_{n}$. This uniquely determines $V_{j}(\mu)$. For minimal outcomes the proof runs analogously.

So let us finally construct the equivalence $x_{-j, n} \mu, w_{n} \sim x_{-j, n} \mu^{\prime}, v_{n}$ with $\mu^{\prime}<\mu$. Take any $\alpha \prec \beta \prec \mu$ with $\alpha$ nonminimal. Define $x:=(\mu, \ldots, \mu, \beta, \ldots, \beta)$, with the $j$ th coordinate the last one equal to $\mu$. If $x_{-j, n} \mu, \alpha \geqslant x_{-j, n} \beta, \beta$, then we find, by restricted solvability, $\mu^{\prime}$ between $\mu$ and $\beta$ to give $x_{-j, n} \mu, \alpha \sim x_{-j, n} \mu^{\prime}, \beta$, and obviously set $\alpha=w_{n}, \beta=v_{n}$. If $x_{-j, n} \mu, \alpha \leqslant x_{-j, n} \beta, \beta$, then we find, by restricted solvability, $w_{n}$ between $\alpha$ and $\beta$ to give $x_{-j, n} \mu, w_{n} \sim x_{-j, n} \beta, \beta$, and obviously set $\beta=\mu^{\prime}=v_{n}$.

Now Observations 15 and 22 give the uniqueness result of Theorem 4(b) also for Case 2.

\section{Completion of the Main Results in Subsection 2.4}

\subsection{Completion of the Proof of Theorems 4(a) and 4(b)}

Since Theorem 4(a) is an immediate consequence of Theorem 4(b), we complete the proof of the latter. The implication (i) $\Rightarrow$ (ii) is straightforward. The implication (ii) $\Rightarrow$ (i) has been established in Section 3 for the case of $n \geqslant 3$ and CI, as well as for the equally spaced case; so have the uniqueness results been for these cases. This 
invoked Example 26 below for the case where $\mathscr{C}$ has exactly two equivalence classes. The densely spaced case with generalized triple cancellation remains to be handled. As pointed out at the definition of CI, generalized triple cancellation implies CI, so for $n \geqslant 3$ the result follows immediately. Actually, with generalized triple cancellation instead of $\mathrm{CI}$, the proof can be considerably shortened since Lemma 13 can then also be obtained if not $z_{1}>z_{n-1}$, so that in the remainder of the proof all the complications about three essential coordinates for $E^{z}$ s, and strict preferences of first over $(n-1)$ th coordinates, can be ignored. See also below, the reasoning below for $n=2$ also applies to the general case of $n \geqslant 2$.

For $n=2$ and the densely spaced case, generalized triple cancellation instead of $\mathrm{CI}$ is necessary and sufficient, in the presence of the other conditions, to give an additive representation on the sets $E^{z}$. See for instance the end of Section 6.2.4 in KLST. Now obviously the requirements that the $E^{z \prime}$ s should have three or more essential coordinates, or that $z_{1}>z_{n-1}$, can and should be omitted everywhere. The work in Lemma 14 can be greatly simplified for $n=2$. Stages 3 to 5 can be omitted. Also the work in Lemma 16 and Lemma 17 can be greatly simplified: if preference between $\left(x_{1}, x_{2}\right)$ and $\left(y_{1}, y_{2}\right)$ is not governed by monotonicity, say $x_{1} \succ y_{1}$ and $x_{2} \prec y_{2}$, then both alternatives are contained in the same $E^{v}$, so that additive representation is direct from the two-dimensional analogue of Lemma 14. Finally, the extension to maximal and minimal outcomes is analogous.

\subsection{Extreme Alternatives: The Proof of Theorem 3}

First we derive the implication (i) $\Rightarrow$ (ii). Transitivity, completeness, and monotonicity are direct. Generalized triple cancellation, hence a fortiori $\mathrm{CI}$, follows straightforwardly, either by elimination of cases, or by substitution of the extended additive representation. The remarkable property of rank-ordered sets is that statement (i) also implies the Archimedean axiom, even if the additive function assigns nonfinite values to extreme alternatives; see Fig. 4. Let there be a minimal outcome $v \cdot V_{1}$ is the only additive value function that may assign value $-\infty$ to $v$. The only problem with the Archimedean axiom that might be expected from this is that there might exist an infinite decreasing standard sequence $\left(\alpha^{k}\right)_{k=1}^{\infty}$ on the first coordinate, with $v$ as lower bound (and $\alpha^{1}$ as upper bound). Say we have $x_{-1, i} \alpha^{k}, v_{i} \sim x_{-1, i} \alpha^{k+1}, w_{i}, k=1, \ldots$, with $w_{i} \succ v_{i}$. The crucial point is that by rankorderedness $w_{i}$ must be a lower bound for the standard sequence, whereas $w_{i}$ cannot be minimal since $v_{i} \prec w_{i}$. By the equality $0<V_{1}\left(\alpha^{1}\right)-V_{1}\left(\alpha^{2}\right)=V_{1}\left(\alpha^{k}\right)-V_{1}\left(\alpha^{k+1}\right)$ for all $k$ and finiteness of $V_{1}\left(w_{i}\right)$ the standard sequence cannot be infinite. Analogously the existence of a maximal outcome which gets assigned value $\infty$ by $V_{n}$ will not lead to an infinite standard sequence.

Next we derive the implication (ii) $\Rightarrow$ (i). By Theorem 4 (b) there exists an additive representation $V$ on the set with the extreme alternatives excluded. In the equally spaced case we can assign to maximal extreme alternatives any value strictly greater than the supremum over the other alternatives, by letting $V_{n}$ assign to the maximal outcome any value strictly greater than the supremum of $V_{n}$ over the nonmaximal outcomes. Analogously, to a minimal alternative any value strictly 


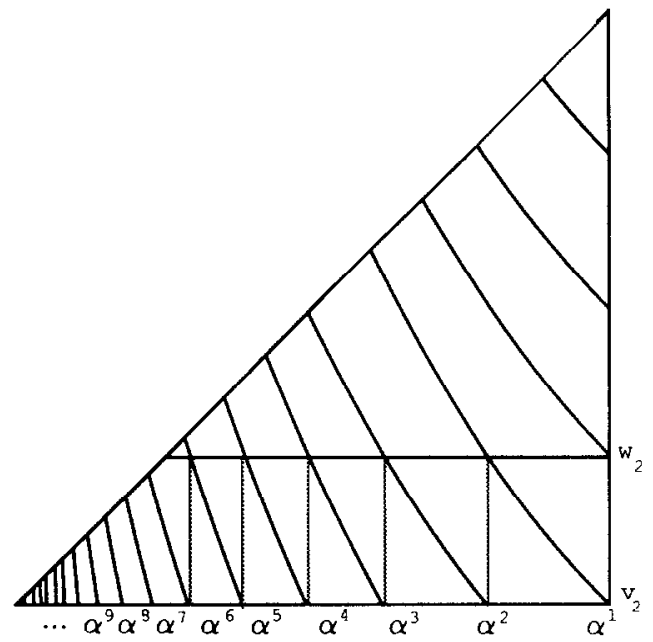

Fig. 4. A counterexample to additive representation on a (rank-ordered) subset of a Cartesian product. Let $n=2, \quad \mathscr{C}=[0,1]$, as in Fig. 1 . Let $\geqslant$ be defined on $\mathscr{C}_{r}^{2}$, and be represented by $\left(x_{1}, x_{2}\right) \mapsto x_{1} \times e^{x_{2}}$. So on $\mathscr{C}_{,}^{2} \backslash\{(0,0)\}$ the preference relation is represented by $\left(x_{1}, x_{2}\right) \mapsto \ln \left(x_{1}\right)+x_{2},(0,0)$ is dispreferred to every other alternative. Curves are equivalence classes; subsequent equivalence classes differ $1 / 4$ as regards the additive representation, so their height difference is $1 / 4$. Also $\ln \left(\alpha^{k}\right)-\ln \left(\alpha^{k+1}\right)$ is $1 / 4$ for all $k$. The curves get steeper and steeper as they get more to the left. Any additive value functions $W_{1}, W_{2}$ would have to satisfy $W_{1}\left(\alpha^{k}\right)-W_{1}\left(\alpha^{k+1}\right)=$ $W_{1}\left(\alpha^{k+1}\right)-W_{1}\left(\alpha^{k+2}\right)$, so that $W_{1}$ would have to assign value $-\infty$ to 0 . On a full Cartesian product this phenomenon would be excluded by the Archimedean axiom; $\left(\alpha^{k}\right)_{k=1}^{k}$ would be a "decreasing" standard sequence with (upper bound 1 and) lower bound 0 . In the present rank-ordered set any decreasing standard sequence is finite since after a finite number of steps $\left(\alpha^{k}, w_{2}\right)$ will cross the border of the rank-ordered set, so the Archimedean axiom is satisfied. The strong Archimedean axiom can be seen to be violated.

smaller than the infimum can be assigned. The reasoning in Subsection 3.1 shows that in particular we can let the range be as described in Corollary 7 . In the densely spaced case we can assign to maximal extreme alternatives any $V$-value greater/ equal the supremum over the other alternatives, by letting $V_{n}$ assign to the maximal outcome any value greater/equal the supremum of $V_{n}$ over the nonmaximal outcomes. Here a supremum value is not taken by nonmaximal outcomes. The supremum may be $\infty$. The case of minimal extreme alternatives is analogous; now we let $V_{1}$ assign to a minimal outcome any value less/equal the infimum over the nonminimal outcomes, which may be $-\infty$. This reasoning also establishes the uniqueness results.

\subsection{The Proof of Theorem 4(c)}

After the definition of the strong Archimedean axiom it was already mentioned that the condition is necessary for additive representability, and that it implies the Archimedean axiom. So let us finally assume that statement (i) holds in the version of Theorem 3 with a maximal outcome with $V_{n}$-value driven to infinity, i.e., the 
supremum of $V_{n}$ over the nonmaximal outcomes is infinite. We show that the strong Archimedean axiom is violated. The case where $V_{1}$ assigns value $-\infty$ to a minimal outcome is analogous.

The equally spaced case is exactly as in Subsection 3.1 ; here actually the usual Archimedean axiom already suffices to obtain a real-valued representation; so infinite values as just supposed will even violate the usual Archimedean axiom. Hence we shall assume that we are in the densely spaced case. We construct an infinite increasing second-order accelerating sequence $\left(\alpha^{k}\right)_{k=1}^{\infty}$, obviously bounded from above by any maximal outcome, and from below by its first outcome. Take any $\left(\alpha^{k}\right)_{k=1}^{\infty}$ such that $V_{n}\left(\alpha^{k+2}\right)-V_{n}\left(\alpha^{k+1}\right)>V_{n}\left(\alpha^{k+1}\right)-V_{n}\left(\alpha^{k}\right)$ for all $k$. Let us fix $k$. By (16) we can take any nonmaximal $\gamma \succ \delta \succ \alpha^{k+2}$ with $V_{1}(\gamma)-V_{1}(\delta)>0$ so small that $V_{n}\left(\alpha^{k+2}\right)-V_{n}\left(\alpha^{k+1}\right)>l\left(V_{1}(\gamma)-V_{1}(\delta)\right)>(l-1)\left(V_{1}(\gamma)-V_{1}(\delta)\right)>$ $V_{n}\left(\alpha^{k+1}\right)-V_{n}\left(\alpha^{k}\right)$ for some $l \in \mathbb{N}$. Set $\beta^{1}:=\alpha^{k}$, and define a standard sequence by equivalences $\left(\gamma, \delta, \ldots, \delta, \beta^{k^{\prime}}\right) \sim\left(\delta, \delta, \ldots, \delta, \beta^{k^{\prime}+1}\right)$. We get $\beta^{\prime}>\alpha^{k+1}$, but there are at least $l \beta$ 's between $\alpha^{k+1}$ and $\alpha^{k+2} .\left(\alpha^{k}\right)_{k=1}^{\infty}$ is indeed a second-order accelerating sequence.

\subsection{The Proof of Proposition 5, Corollary 6, and Corollary 7}

Corollary 7, and Corollary 6 and Proposition 5 for the equally spaced case, follow from the analysis in Subsections 3.1 and 4.2. For the densely spaced case we saw that the extended additive representing function can and should assign to each maximal alternative any value greater/equal the supremum over the nonmaximal alternatives. Analogous results hold for minimal alternatives. This implies Proposition 5. For Corollary 6 note that, if a maximal outcome exists, then $V_{2}$ will assign a real value to it so is bounded above. Under proportionality the other additive value functions, in particular $V_{1}$, are bounded above as well. The case of a minimal outcome is analogous. Corollary 6 now follows from Proposition 5.

\section{ReMARKS AND EXAMPLES}

In this section we give some examples and elucidations.

Remark 23 [Thomsen Condition]. For the case $n=2$ we could have replaced triple cancellation above by the "Thomsen condition" (see KLST); the usual independence condition accompanying these for the case of $n=2$ is implied by monotonicity. In the presence of the other conditions the Thomsen condition, as well as triple cancellation, is equivalent to the existence of an additive representation on full Cartesian products, thus on the sets $E^{z}$; that was the only implication of triple cancellation needed in the above theorems.

Remark 24 [Absence of Tradeoffs for Extreme Outcomes]. The proof in Subsection 4.2 already showed complications that may be expected for extreme outcomes. On rank-ordered subsets the Archimedean axiom does not prevent the 
additive representation from being "driven to infinity." This is illustrated in Example 25 below.

The reason lies in the shape of the particular subset of a Cartesian product that we consider, i.e., the subset of rank-ordered alternatives. If $x_{n}=\mu$ for $\mu$ maximal, then we must have $x_{j} \sim \mu$ for all $j$; if $x_{1}=v$ for $v$ minimal, then we must have $x_{j} \sim v$ for all $j$. To find out about the difference $V_{n}(\mu)-V_{n}(\alpha)$ for any $\alpha<\mu$ in usual additive representation one considers the preference between $x_{-n} \alpha$ and $y_{-n} \mu$ for $\sum_{j=1}^{n \cdots 1}\left(V_{j}\left(x_{j}\right)-V_{j}\left(y_{j}\right)\right)>0$, to compare $\sum_{j=1}^{n-1}\left(V_{j}\left(x_{j}\right)-V_{j}\left(y_{j}\right)\right)$ with $V_{n}(\mu)-V_{n}(\alpha)$. However, in the present rank-ordered context $y_{-n} \mu$ is available only if $y_{j} \geqslant \mu$ for all $j$, i.e., each $y_{j}$ must be maximal; the latter excludes $\sum_{j=1}^{n-1}\left(V_{j}\left(x_{j}\right)-V_{j}\left(y_{j}\right)\right)>0$. Loosely speaking, maximal outcomes on the $n$th coordinate are never involved in tradeoffs, neither are minimal outcomes on the first coordinate. This is the reason that for an additive value function $V_{n}$ the value $V_{n}(\mu)$ can be arbitrarily increased, and that in some cases an additive value function $V_{n}$ may have to assign value $\infty$ to $\mu$.

\section{EXAMPLE 25 [Extreme Alternatives].}

EXAMPLe 25a (Only lower/upper bound at maximal/minimal extreme alternatives). This example shows that for additive representations on a rank-ordered subset, the value at the maximal extreme alternatives of the rank-ordered subset is not bounded from above; it can be any value higher than some lower bound. Analogously the value at the minimal extreme alternatives of the rank-ordered subset is not bounded from below, and can be any value lower than some upper bound; so uniqueness results differ from the usual ones on full Cartesian products.

Let $\mathscr{C}=[0,1]$. Let $V_{j}$ be identity for all $j .\left(V_{j}\right)_{j=1}^{n}$ are additive value functions for $\succcurlyeq$ on $\mathscr{C}_{r}^{n}$. $\succcurlyeq$ is a weak order, it satisfies generalized triple cancellation and hence CI, restricted solvability, and the strong Archimedean axiom which implies the Archimedean axiom. Any additive representation $W$ of $\geqslant$ will on $\mathscr{C}_{r}^{n} \backslash\{(0, \ldots, 0),(1, \ldots, 1)\}$ be of the form $W=\tau+\sigma V$ for real $\tau$ and positive $\sigma$. Further $W_{1}(0)$ can be any value $\leqslant \tau+\sigma V_{1}(0)$, and $W_{n}(1)$ can be any value $\geqslant \tau+\sigma V_{n}(1)$.

EXAmPLE 25b (Value driven to $-\infty$ at minimal extreme alternatives). See Fig. 4 for the two-dimensional case. This example shows that for additive representations on a rank-ordered subset, the value at the minimal elements of the rank-ordered subset may be forced to be $-\infty$, so that strictly speaking there does not exist an additive representation.

Let $\mathscr{C}=[0,1]$. Let $V_{j}$ be identity for all $j>1$. For $j=1$, on $\left.] 0,1\right], V_{1}$ is $\ln . V_{1}(0)$ is left undefined. (Setting $V_{1}(0):=-\infty$ would give an extended additive representation.) On $\mathscr{C}_{r}^{n} \backslash\{(0, \ldots, 0)\},\left(V_{j}\right)_{j=1}^{n}$ are additive value functions for $\succcurlyeq$. Obviously, $(0, \ldots, 0) \prec x$ for all $x \neq(0, \ldots, 0)$. $\geqslant$ is a weak order, satisfies generalized triple cancellation and hence CI, satisfies restricted solvability, and will at the end of this example be shown to satisfy the Archimedean axiom. It does not satisfy the strong 
Archimedean axiom. For any additive representation $W$ of $\geqslant$, on $\mathscr{C} \backslash\{0\}$ the function $W_{1}$ will be of the form $\tau+\sigma V_{1}$ for real $\tau$ and positive $\sigma$. Hence there does not exist an additive representation on the entire $\mathscr{C}_{r}^{n} ; W_{1}(0)$ would have to be $-\infty$, i.e., smaller than any real number.

Let us now show that the Archimedean axiom nevertheless holds. See again Fig. 4. It is easy to see that a bounded standard sequence $\alpha^{1}, \alpha^{2}, \ldots$, must be finite if it is on coordinate 2 or higher, and also that it must be finite if it is increasing $\left(\alpha^{k+1}>\alpha^{k}\right)$ on coordinate 1 . So let $x_{-1, i} \alpha^{k}, v_{i} \sim x_{-1, i} \alpha^{k+1}, w_{i}$ for all $k=1,2, \ldots$, with all involved alternatives contained in $X$, and $v_{i} \prec w_{i}$ to have the standard sequence decreasing. We have $w_{i}>v_{i}$ so $w_{i}>0$; substituting the additive value functions shows that $\forall k: \ln \left(\alpha^{k}\right)-\ln \left(\alpha^{k+1}\right)=w_{i}-v_{i}$. The crucial point is that every involved alternative must be rank-ordered, so that $\alpha^{k} \geqslant w_{i}$ for all $k$. Hence the length of the standard sequence cannot be larger than $1-\ln \left(w_{i}\right) /\left(w_{i}-v_{i}\right)$.

Obviously, there do exist infinite bounded second-order accelerating sequences. For example, such a sccond-order accelcrating sequence $\left(\alpha^{k}\right)_{k=1}^{\infty}$ can be defined by $\ln \left(\alpha^{k}\right)-\ln \left(\alpha^{k+1}\right)=w_{i}-v_{i}$ for all $k$, with, say, $\alpha^{1}=1, v_{i}=0, w_{i}=\frac{1}{4}$. For each $\alpha^{k+2}$, $\alpha^{k+1}, \alpha^{k}$ some $0<v_{2}^{\prime}<w_{2}^{\prime}<\alpha^{k+2}$ can be found to give, loosely speaking, a "measure stick" on coordinate 2 such that for some integer $l, \ln \left(\alpha^{k}\right)-\ln \left(\alpha^{k+1}\right)=$ $\ln \left(\alpha^{k+1}\right)-\ln \left(\alpha^{k+2}\right)=l\left(w_{2}^{\prime}-v_{2}^{\prime}\right)$. Equivalences involving appropriate outcomes between $\alpha^{k+2}$ and $\alpha^{k}$ show that the $\alpha^{k}$ s form a second-order accelerating sequence. Actually this can be called a second-order standard sequence.

EXAmpLe 25c (Value driven to $\infty$ at maximal extreme alternatives). This example shows that for additive representations on a rank-ordered subset, the value at the maximal elements of the rank-ordered subset may be forced to be $\infty$, so that there does not exist an additive representation in the usual sense.

We vary with respect to Example $25 \mathrm{~b}$ by setting $V_{1}=\cdots=V_{n-1}=$ identity, $V_{n}: \alpha \mapsto 1 /(1-\alpha)$ on $\left[0,1 \Gamma, V_{n}(1)\right.$ undefined. (Setting $V_{1}(1):=\infty$ would give an extended additive representation.) On $\mathscr{C}_{r}^{n} \backslash\{(1, \ldots, 1)\},\left(V_{j}\right)_{j=1}^{n}$ are additive value functions for $\geqslant$, further $(1, \ldots, 1)>x$ for all $x \in \mathscr{C}_{r}^{n} \backslash\{(1, \ldots, 1)\} . \geqslant$ is a weak order, satisfies generalized triple cancellation and hence CI, satisfies restricted solvability, and can be seen to satisfy the Archimedean axiom, analogously to Example 25b. Now any increasing standard sequence on coordinate $n$ can be seen to be finite since it cannot exceed a $w_{i}<1$ because of rank-orderedness. For any additive representation $W$ of $\geqslant$, on $\mathscr{C} \backslash\{1\}$ the function $W_{n}$ will be of the form $\tau+\sigma V_{n}$ for real $\tau$ and positive $\sigma$. Hence there does not exist an additive representation on the entire $\mathscr{C}_{r}^{n} ; W_{n}(1)$ would have to be $\infty$, i.e., larger than any real number. Again, the strong Archimedean axiom can be seen to be violated.

Let us comment more on Examples $25 \mathrm{~b}$ and $25 \mathrm{c}$. Additive representation is equivalent to multiplicative representation with positive real numbers. The case where an additive value function is driven to $-\infty$, as in Example 25b, can be modeled multiplicatively by taking exponent; e.g., set $\left(x_{1}, \ldots, x_{n}\right) \mapsto x_{1} \times$ $e^{x_{2}} \times \cdots \times e^{x_{n}}$ in Example 25b. So the additive value $-\infty$ is replaced by the 
multiplicative value 0 . The case where an additive value function is driven to $\infty$, as in Example $25 \mathrm{c}$, can also be modeled multiplicatively, by taking minus exponent of minus the additive representation. Then the additive value $\infty$ is replaced by the multiplicative value 0 . However, it may also happen that simultaneously the additive value function $V_{1}$ is forced to be $-\infty$ at a minimal outcome, and the additive value function $V_{n}$ is forced to be $\infty$ at a maximal outcome. Then no multiplicative representation exists.

Next we turn to the case where $\mathscr{C}$ has exactly two equivalence classes.

Example 26 [Two Equivalence Classes of Outcomes]. Let $\mathscr{C}=\mathscr{C}_{+} \cup \mathscr{C}_{-}$. All elements of $\mathscr{C}_{+}$are $\sim$ '-equivalent, as well as all elements of $\mathscr{C}_{-}$; each element of $\mathscr{C}_{+}$ is strictly preferred to each element of $\mathscr{C}_{-} . X$ consists of all elements of $\mathscr{C}^{n}$ that have all coordinates up to some coordinate $j,(0<j<n$ if extreme alternatives are not included, $0 \leqslant j \leqslant n$ if they are) in $\mathscr{C}_{+}$, and all subsequent coordinates in $\mathscr{C}_{-}$. Let $\succcurlyeq$ be a weak order on $X$. Monotonicity uniquely determines $\geqslant$ as follows. If, for two elements of $X, j$ as described above is identical, the elements are equivalent, in the other case the element with the larger $j$ is strictly preferred. All conditions of statement (ii) of Theorems $4(\mathrm{~b}), 4(\mathrm{c})$, and 3 are satisfied, as well as CI and restricted solvability. $\left(V_{j}\right)_{j=1}^{n}$ are additive value functions if and only if every $V_{j}$ is constant on $\mathscr{C}_{+}$, and is "smaller"-constant on $\mathscr{C}_{-}$. So any $V_{j}$ is an ordinal (=interval) scale, and can be transformed independently of the other additive value functions. $V$ is an ordinal scale. This implies that $V$ is an interval scale if and only if its range has no more than two elements, which holds true if and only if $X=\mathscr{C}_{r}^{2} \backslash\{$ extreme alternatives $\}$ and $n \leqslant 3$.

\section{CONCLUSION}

Additive representation results have been provided for rank-ordered subsets of Cartesian products. Contrary to what has often been thought, these results show characteristics different from additive representation on full Cartesian products, and solid proofs are more complicated than has usually been supposed.

\section{ACKNOWLEDGMENTS}

Discussions with Alain Chateauneuf initiated this research. Peter Fishburn and an anonymous referee gave helpful comments; the latter posed the question how to strengthen the usual Archimedean axiom to get a real-valued representation.

\section{REFERENCES}

Allais, M. (1988). The general theory of random choices in relation to the invariant cardinal utility function and the specific probability function. In B. R. Munier (Ed.), Risk, Decision and Rationality (pp. 231-289). Dordrecht: Reidel. 
BeCKer, J. L., \& SARIN, R. K. (1989). Economics of ambiguity. Duke University, Fuqua School of Business, Durham, NC.

Chateauneuf, A. (1990a). On the use of non-additive probabilities in modeling uncertainty aversion and risk aversion, Journal of Mathematical Economics, to appear.

Chateauneuf, A. (1990b). On the use of comonotonicity in the axiomatization of EURDP theory for arbitrary consequences. CERMSEM, University of Paris I; paper presented at Fifth International Conference on the Foundations and Applications of Utility, Risk and Decision Theory (FUR-90).

CHEw, S. H. (1989). An axiomatic generalization of the quasilinear mean and Gini mean with application to decision theory. Annals of Statistics, tentatively accepted; rewritten vesion of Chew, S. H. (1985). An axiomatization of the rank-dependent quasilinear mean generalizing the Gini mean and the quasilinear mean (Economics Working Paper No. 156). Baltimore, MD: Johns Hopkins University.

Chew, S. H., \& EpSTEIN, L. G. (1989a). Axiomatic rank-dependent means. Annals of Operations Research 19, 299-309.

Chrw, S. H., \& EPSTEIN, L. G. (1989b). A unifying approach to axiomatic non-expected utility theories. Journal of Economic Theory, 49, 207-240.

Doignon, J. P., \& Falmagne, J. C. (1974). Difference measurement and simple scalability with restricted solvability. Jornal of Mathematical Psychology, 11, 473-499.

EBERT, U. (1988a). Measurement of inequality: An attempt at unification and generalization. Social Choice and Welfare, 5, 147-169.

EBERT, U. (1988b). Rawls and Bentham reconciled, Theory and Decision, 24, 215-223.

FishBURN, P. C. (1976). Utility independence on subsets of product sets. Operations Research, 24, 245-255.

GILBOA, I. (1987). Expected utility with purely subjective non-additive probabilities. Journal of Mathematical Economics, 16, 65-88.

GILBOA, I. (1989). Expectations and variations in multi-period decisions. Econometrica, 57, 1153-1169.

GrEeN, J. \& JUlLIEN, B. (1988). Ordinal independence in non-linear utility theory. Journal of Risk and Uncertainty. 1, 355-387.

HöHLE, U. (1982, January). Integration with respect to fuzzy measures. Proceedings IFAC Symposium on Theory and Application of Digital Control, New Delhi, 35-37.

Jafrray, J. Y. (1974a). Existence, Propriétés de Continuité, Additivité de Fonctions d'Utilité sur un Espace Partiellement ou Totalement Ordonné. Ph.D. dissertation, Université de Paris VI, Paris.

JAFFRAY, J. Y. (1974b). On the extension of additive utilities to infinite sets. Journal of Mathematical Psychology, 11, 431-452.

Krantz, D. H., LuCE, R. D., Suppes, P., \& TVERSky, A. (1971). Foundations of measurement (Vol. I.) Additive and polynomial representations. New York: Academic Press.

LUCE, R. D. (1988). Rank-Dependent, subjective expected-utility representations. Journal of Risk and Uncertainty, 1, 305-332.

LUCE, R. D., \& NARENS, L. (1985). Classification of concatenation measurement structures according to scale type. Journal of Mathematical Psychology, 29, 1-72.

Miyamoto, J. M. (1988). Generic utility theory: Measurement foundations and applications in multiattribute utility theory. Journal of Mathematical Psychology, 32, 357-404.

Murofushi, T., \& Sugeno, M. (1989). An interpretation of fuzzy measures and the Choquet integral as an integral with respect to a fuzzy measure. Fuzzy Sets and Systems, 29, 201-227.

NaKamura, Y. (1990a). Subjective expected utility with non-additive probabilities on finite state space. Journal of Economic Theory, 51, 346-366.

Nakamura, Y. (1990b). Multi-symmetric structures and non-expected utility. Journal of Mathematical Psychology, to appear.

Pfanzagl, J. (1968). Theory of measurement. Vienna: Physica-Verlag.

QUigGIN, J. (1982). A theory of anticipated utility. Journal of Economic Behaviour and Organization, 3, 323-343.

QuigGIN, J. (1989). Sure things-Dominance and independence rules for choice under uncertainty. Annals of Operations Research, 19, 335-357. 
SCHMEIDLER, D. (1989). Subjective probability and expected utility without additivity. Econometrica, 57, 571-587.

Scotr, D. (1964). Measurement structures and linear inequalities. Journal of Mathematical Psychology, $1,233-247$.

SEGAL, U. (1989). Anticipated utility: A measure representation approach. Annals of Operations Research, 19, 359-373.

SEGAL, U. (1990). Additively separable representations on non-convex sets (Manuscript). Department of Economics, University of Toronto, Canada.

WAKKER, P. P. (1986). Representations of choice situations. Ph.D. dissertation, Department of Economics, University of Brabant, The Netherlands.

WAKKER, P. P. (1988). The algebraic versus the topological approach to additive representations. Journal of Mathematical Psychology, 32, 421-435.

WAKKER, P. P. (1989a). Continuous subjective expected utility with nonadditive probabilities. Journal of Mathematical Economics, 18, 1-27.

WAKKER, P. P. (1989b). Additive representations of preferences: A new foundation of decision analysis. Dordrecht: Kluwer Academic Publishers (formerly Reidel).

WAKKER, P. P. (1989c). From finite- to infinite-dimensional integral representations; unbounded utility for Savage (1954) and others. Mathematics of Operations Research, accepted subject to revision.

Wakker, P. P. (1990a). A behavioral foundation for fuzzy measures. Fuzzy Sets and Systems, 37. 327-350.

WaKkeR, P. P. (1990b). Under stuchastic dominance Choquet-expected utility and anticipated utility are identical. Theory and Decision, 29, 119-132.

WAKKER, P. P. (1990c). Additive representation for equally spaced structures. Journal of Mathematical Psychology, to appear.

WAKKFR, P. P. (1990d). Counterexamples to additive representation on nonfull Cartesian products. Duke University, Fuqua School of Business, Durham, NC.

WAKKer, P. P. (1990e). Additive representations on rank-ordered sets. Part II. The topological approach. Journal of Mathematical Economics, accepted under condition of revision.

WAKKER, P. P. (1990f). Continuity, absolute continuity, and weak convergence in anticipated-utility representations. Duke University, Fuqua School of Business, Durham, NC.

Weymark, J. A. (1981). Generalized Gini inequality indices. Mathematical Social sciences, 1, 409-430.

YAARI, M. E. (1987). The dual theory of choice under risk. Econometrica, 55, 95-115.

YAGER, R. R. (1988). On ordered weighted averaging aggregation operators in multicriteria decisionmaking. IEtE Iransactions on Systems, Man, and Cybernetics, 18, 183-190.

RECEIVED: February 12, 1990 